Open Access

\title{
An original phylogenetic approach identified mitochondrial haplogroup T1a1 as inversely associated with breast cancer risk in BRCA2 mutation carriers
}

Sophie Blein ${ }^{1,2,3}$, Claire Bardel 2,3,4, Vincent Danjean ${ }^{5,6}$, Lesley McGuffog ${ }^{7}$, Sue Healey ${ }^{8}$, Daniel Barrowdale ${ }^{7}$, Andrew Lee ${ }^{7}$, Joe Dennis ${ }^{7}$, Karoline B Kuchenbaecker ${ }^{7}$, Penny Soucy ${ }^{9}$, Mary Beth Terry ${ }^{10}$, Wendy K Chung ${ }^{11,12}$, David E Goldgar ${ }^{13}$, Saundra S Buys ${ }^{14}$, Breast Cancer Family Registry ${ }^{15}$, Ramunas Janavicius ${ }^{16,17}$, Laima Tihomirova ${ }^{18}$, Nadine Tung ${ }^{19}$, Cecilia M Dorfling ${ }^{20}$, Elizabeth J van Rensburg ${ }^{20}$, Susan L Neuhausen ${ }^{21}$, Yuan Chun Ding ${ }^{21}$, Anne-Marie Gerdes ${ }^{22}$, Bent Ejlertsen ${ }^{23}$, Finn C Nielsen ${ }^{24}$, Thomas VO Hansen ${ }^{24}$, Ana Osorio ${ }^{25,26}$, Javier Benitez ${ }^{25,26}$, Raquel Andrés Conejero ${ }^{27}$, Ena Segota ${ }^{28,171}$, Jeffrey N Weitzel ${ }^{29}$, Margo Thelander ${ }^{30}$, Paolo Peterlongo ${ }^{31}$, Paolo Radice ${ }^{32}$, Valeria Pensotti ${ }^{29,33}$, Riccardo Dolcetti ${ }^{34}$, Bernardo Bonanni ${ }^{35}$, Bernard Peissel ${ }^{36}$, Daniela Zaffaroni ${ }^{36}$, Giulietta Scuvera $^{36}$, Siranoush Manoukian ${ }^{36}$, Liliana Varesco ${ }^{37}$, Gabriele L Capone ${ }^{38,39}$, Laura Papi ${ }^{39}$, Laura Ottini ${ }^{40}$, Drakoulis Yannoukakos ${ }^{41}$, Irene Konstantopoulou ${ }^{42}$, Judy Garber ${ }^{43}$, Ute Hamann ${ }^{44}$, Alan Donaldson ${ }^{45}$, Angela Brady ${ }^{46}$, Carole Brewer ${ }^{47}$, Claire Foo ${ }^{48}$, D Gareth Evans ${ }^{49}$, Debra Frost ${ }^{50}$, Diana Eccles ${ }^{51}$, EMBRACE $^{50}$, Fiona Douglas ${ }^{52}$, Jackie Cook ${ }^{53}$, Julian Adlard ${ }^{54}$, Julian Barwell ${ }^{55}$, Lisa Walker ${ }^{56}$, Louise Izatt ${ }^{57}$, Lucy E Side ${ }^{58}$, M John Kennedy ${ }^{58,59,60}$, Marc Tischkowitz ${ }^{61}$, Mark T Rogers ${ }^{62}$, Mary E Porteous ${ }^{63}$, Patrick J Morrison ${ }^{64,65}$, Radka Platte ${ }^{50}$, Ros Eeles ${ }^{66}$, Rosemarie Davidson ${ }^{67}$, Shirley Hodgson ${ }^{68}$, Trevor Cole ${ }^{69}$, Andrew K Godwin ${ }^{70}$, Claudine Isaacs $^{71}$, Kathleen Claes ${ }^{72}$, Kim De Leeneer ${ }^{72}$, Alfons Meindl ${ }^{73}$, Andrea Gehrig ${ }^{74}$, Barbara Wappenschmidt ${ }^{75,76}$, Christian Sutter ${ }^{77}$, Christoph Engel ${ }^{78}$, Dieter Niederacher ${ }^{79}$, Doris Steinemann ${ }^{80}$, Hansjoerg Plendl ${ }^{81}$, Karin Kast ${ }^{82}$, Kerstin Rhiem ${ }^{75,76}$, Nina Ditsch ${ }^{73}$, Norbert Arnold ${ }^{83}$, Raymonda Varon-Mateeva ${ }^{84}$, Rita K Schmutzler ${ }^{75,76,85}$, Sabine Preisler-Adams ${ }^{86^{\wedge}}$, Nadja Bogdanova Markov ${ }^{86}$, Shan Wang-Gohrke ${ }^{87}$, Antoine de Pauw ${ }^{88}$, Cédrick Lefol $^{88}$, Christine Lasset ${ }^{4,89}$, Dominique Leroux ${ }^{90,91}$, Etienne Rouleau ${ }^{92}$, Francesca Damiola ${ }^{1}$, GEMO Study Collaborators, Hélène Dreyfus ${ }^{90,91}$, Laure Barjhoux¹ , Lisa Golmard ${ }^{88}$, Nancy Uhrhammer ${ }^{93}$, Valérie Bonadona ${ }^{4,89}$, Valérie Sornin ${ }^{1}$, Yves-Jean Bignon ${ }^{93}$, Jonathan Carter ${ }^{94}$, Linda Van Le ${ }^{95}$, Marion Piedmonte ${ }^{96}$, Paul A DiSilvestro ${ }^{97}$, Miguel de la Hoya ${ }^{98}$, Trinidad Caldes ${ }^{98}$, Heli Nevanlinna ${ }^{99}$, Kristiina Aittomäki ${ }^{100}$, Agnes Jager ${ }^{101}$, Ans MW van den Ouweland ${ }^{102}$, Carolien M Kets $^{103}$, Cora M Aalfs ${ }^{104}$, Flora E van Leeuwen ${ }^{105}$, Frans BL Hogervorst ${ }^{106}$, Hanne EJ Meijers-Heijboer ${ }^{107}$, HEBON, Jan C Oosterwijk ${ }^{108}$, Kees EP van Roozendaal ${ }^{109}$, Matti A Rookus ${ }^{105}$, Peter Devilee ${ }^{110,111}$, Rob B van der Luijt ${ }^{112}$, Edith Olah ${ }^{113}$, Orland Diez ${ }^{114}$, Alex Teulé ${ }^{115}$, Conxi Lazaro ${ }^{116}$, Ignacio Blanco ${ }^{115}$, Jesús Del Valle ${ }^{116}$, Anna Jakubowska ${ }^{117}$, Grzegorz Sukiennicki ${ }^{117}$, Jacek Gronwald ${ }^{117}$, Jan Lubinski ${ }^{117}$, Katarzyna Durda ${ }^{117}$, Katarzyna Jaworska-Bieniek ${ }^{117}$, Bjarni A Agnarsson ${ }^{118}$, Christine Maugard ${ }^{119}$, Alberto Amadori ${ }^{120,121}$, Marco Montagna ${ }^{121}$, Manuel R Teixeira ${ }^{122,123}$, Amanda B Spurdle ${ }^{8}$, William Foulkes ${ }^{124}$, Curtis Olswold ${ }^{125}$, Noralane M Lindor ${ }^{126}$, Vernon S Pankratz ${ }^{125}$, Csilla I Szabo ${ }^{127}$, Anne Lincoln ${ }^{128}$, Lauren Jacobs ${ }^{128}$, Marina Corines ${ }^{128}$, Mark Robson ${ }^{129}$, Joseph Vijai ${ }^{129}$,

\footnotetext{
* Correspondence: david.cox@lyon.unicancer.fr

Deceased

'INSERM U1052, CNRS UMR5286, Université Lyon 1, Centre de Recherche en Cancérologie de Lyon, Lyon, France

${ }^{2}$ Université de Lyon, 69000 Lyon, France

Full list of author information is available at the end of the article
} 
Andreas Berger ${ }^{130}$, Anneliese Fink-Retter ${ }^{130}$, Christian F Singer ${ }^{130}$, Christine Rappaport ${ }^{130}$, Daphne Geschwantler Kaulich ${ }^{130}$, Georg Pfeiler ${ }^{130}$, Muy-Kheng Tea ${ }^{130}$, Mark H Greene ${ }^{131}$, Phuong L Mai ${ }^{131}$, Gad Rennert ${ }^{35,132,133}$, Evgeny N Imyanitov ${ }^{134}$, Anna Marie Mulligan ${ }^{135,136}$, Gord Glendon ${ }^{137,138}$, Irene L Andrulis ${ }^{135,138,139}$, Sandrine Tchatchou ${ }^{138}$, Amanda Ewart Toland ${ }^{140,141,142,143}$, Inge Sokilde Pedersen ${ }^{144}$, Mads Thomassen ${ }^{145}$, Torben A Kruse ${ }^{145}$, Uffe Birk Jensen ${ }^{146}$, Maria A Caligo ${ }^{147}$, Eitan Friedman ${ }^{148}$, Jamal Zidan ${ }^{149}$, Yael Laitman ${ }^{148}$, Annika Lindblom ${ }^{150}$, Beatrice Melin ${ }^{151}$, Brita Arver ${ }^{152}$, Niklas Loman ${ }^{153}$, Richard Rosenquist ${ }^{154}$, Olufunmilayo I Olopade ${ }^{155}$, Robert L Nussbaum ${ }^{156}$, Susan J Ramus ${ }^{157}$, Katherine L Nathanson ${ }^{158}$, Susan M Domchek ${ }^{158}$, Timothy R Rebbeck ${ }^{159}$, Banu K Arun ${ }^{160}$, Gillian Mitchell ${ }^{161,162}$, Beth Y Karlan ${ }^{163}$, Jenny Lester ${ }^{163}$, Sandra Orsulic ${ }^{163}$, Dominique Stoppa-Lyonnet ${ }^{88,164,165}$, Gilles Thomas ${ }^{166,167^{\wedge}}$, Jacques Simard ${ }^{9}$, Fergus J Couch ${ }^{125,168}$, Kenneth Offit ${ }^{129}$, Douglas F Easton', Georgia Chenevix-Trench ${ }^{8}$, Antonis C Antoniou', Sylvie Mazoyer ${ }^{1,2,3}$, Catherine M Phelan ${ }^{169}$, Olga M Sinilnikova ${ }^{1,2,3,170^{\wedge}}$ and David G Cox ${ }^{1,2,3^{*}}$

\begin{abstract}
Introduction: Individuals carrying pathogenic mutations in the BRCA1 and BRCA2 genes have a high lifetime risk of breast cancer. BRCA1 and BRCA2 are involved in DNA double-strand break repair, DNA alterations that can be caused by exposure to reactive oxygen species, a main source of which are mitochondria. Mitochondrial genome variations affect electron transport chain efficiency and reactive oxygen species production. Individuals with different mitochondrial haplogroups differ in their metabolism and sensitivity to oxidative stress. Variability in mitochondrial genetic background can alter reactive oxygen species production, leading to cancer risk. In the present study, we tested the hypothesis that mitochondrial haplogroups modify breast cancer risk in BRCA1/2 mutation carriers.
\end{abstract}

Methods: We genotyped 22,214 (11,421 affected, 10,793 unaffected) mutation carriers belonging to the Consortium of Investigators of Modifiers of BRCA1/2 for 129 mitochondrial polymorphisms using the iCOGS array. Haplogroup inference and association detection were performed using a phylogenetic approach. ALTree was applied to explore the reference mitochondrial evolutionary tree and detect subclades enriched in affected or unaffected individuals.

Results: We discovered that subclade T1a1 was depleted in affected BRCA2 mutation carriers compared with the rest of clade $T$ (hazard ratio $(\mathrm{HR})=0.55 ; 95 \%$ confidence interval $(C \mathrm{Cl}), 0.34$ to $0.88 ; P=0.01)$. Compared with the most frequent haplogroup in the general population (that is, $\mathrm{H}$ and T clades), the T1a1 haplogroup has a HR of $0.62(95 \% \mathrm{Cl}, 0.40$ to $0.95 ; P=0.03$ ). We also identified three potential susceptibility loci, including G13708A/rs28359178, which has demonstrated an inverse association with familial breast cancer risk.

Conclusions: This study illustrates how original approaches such as the phylogeny-based method we used can empower classical molecular epidemiological studies aimed at identifying association or risk modification effects.

\section{Introduction}

Breast cancer is a multifactorial disease with genetic, lifestyle and environmental susceptibility factors. Approximately $15 \%$ to $20 \%$ of the familial aggregation of breast cancer is accounted for by mutations in high-penetrance susceptibility genes [1-3], such as BRCA1 and BRCA2. Pathogenic mutations in BRCA1 and BRCA2 confer lifetime breast cancer risk of $60 \%$ to $85 \%[4,5]$ and $40 \%$ to $85 \%[4,5]$, respectively. Other genomic variations (for example, in genes encoding proteins interacting with BRCA1 and BRCA2) have been identified as modifiers of breast cancer risk and increase or decrease the risk initially conferred by $B R C A 1$ or $B R C A 2$ mutation [6].

$B R C A 1$ and $B R C A 2$ are involved in DNA repair mechanisms, including double-strand break (DSB) repair by homologous recombination $[7,8]$. DSBs are considered to be among the most deleterious forms of DNA damage because the integrity of both DNA strands is compromised simultaneously. These breaks can lead to genomic instability resulting in translocations, deletions, duplications or mutations when not correctly repaired [9]. Reactive oxygen species (ROS) are one of the main causes of DSBs, along with exposure to ionizing radiation, various chemical agents and ultraviolet light [10].

ROS are naturally occurring chemical derivatives of metabolism. Elevated levels of ROS and downregulation of ROS scavengers and/or antioxidant enzymes can lead to oxidative stress, which is associated with a number of human diseases, including various cancers [11]. The electron transport chain process, which takes place in the mitochondria, generates the majority of ROS in human cells. Variations in the mitochondrial genome have been shown to be associated with metabolic phenotypes and 
oxidative stress markers [12]. Mitochondrial dysfunction recently was shown to promote breast cancer cell migration and invasion through the accumulation of a transcription factor, hypoxia-inducible factor $1 \alpha$, via increased production of ROS [13].

Human mitochondrial DNA (mtDNA) has undergone a large number of mutations that have segregated during evolution. Those changes are now used to define mitochondrial haplogroups. Some of these changes slightly modify metabolic performance and energy production; thus, not all haplogroups have identical metabolic capacities [14]. It has been hypothesized that the geographic distribution of mitochondrial haplogroups results from selection of metabolic capacities driven mainly by adaptation to climate and nutrition $[15,16]$.

Mitochondrial haplogroups have been associated with diverse multifactorial diseases, such as Alzheimer's disease [17], hypertrophic cardiomyopathy [18], retinal diseases [19] or age-related macular degeneration [20]. Variations in mtDNA have also been linked to several types of cancer, such as gastric cancer [21] or renal cell carcinoma [22]. Interestingly, variations in mtDNA have been linked to several types of female cancers, including endometrial [23], ovarian [24] and breast cancer [25,26]. A recent study underlined the possibility that mtDNA might be involved in the pathogenic and molecular mechanisms of familial breast cancer [27].

The Collaborative Oncological Gene-environment Study [28] (COGS) is a European project designed to improve understanding of genetic susceptibility to breast, ovarian and prostate cancer. This project involves several consortia: the Breast Cancer Association Consortium (BCAC) [29], the Ovarian Cancer Association Consortium [30], the Prostate Cancer Association Group to Investigate Cancer Associated Alterations in the Genome (PRACTICAL) [31] and the Consortium of Investigators of Modifiers of BRCA1/2 (CIMBA) [32]. CIMBA is a collaborative group of researchers working on genetic modifiers of cancer risk in BRCA1 and BRCA2 mutation carriers. As part of the COGS project, more than 200,000 single-nucleotide polymorphisms (SNPs) were genotyped for BRCA1 and BRCA2 female mutation carriers on the iCOGS chip, including 129 mitochondrial polymorphisms. The iCOGS chip is a custom Illumina ${ }^{\text {Tx }}$ Infinium genotyping array (Illumina, San Diego, CA, USA) designed to test, in a cost-effective manner, genetic variants related to breast, ovarian and prostate cancers.

In this study, we explored mitochondrial haplogroups as potential modifiers of breast cancer risk in women carrying pathogenic $B R C A 1$ or $B R C A 2$ mutations. Our study includes females diagnosed with breast cancer and unaffected carriers belonging to CIMBA. We used an original analytic phylogenetics-based approach implemented in a homemade algorithm and in the program ALTree
$[33,34]$ to infer haplogroups and to detect associations between haplogroups and breast cancer risk.

\section{Methods}

Ethics statement

A signed informed written consent form was obtained from all participants. All contributing studies involved in CIMBA received approvals from the institutional review committees at their host institutions. Ethical committees that approved access to the data analyzed in this study are listed in Additional file 1.

\section{BRCA1 and BRCA2 mutation carriers}

Final analyses included 7,432 breast cancer cases and 7,104 unaffected BRCA1 mutation carriers, as well as 3,989 invasive breast cancer and 3,689 unaffected BRCA2 mutation carriers, all belonging to CIMBA. Supplementary specifications regarding inclusion profiles and studies belonging to CIMBA are available in the reports by Couch et al. [35] and Gaudet et al. [36]. All analyses were conducted separately on CIMBA BRCA1 and BRCA2 mutation carriers (abbreviated pop1 and pop2, respectively). Eligible female carriers were aged 18 years or older and had a pathogenic mutation in BRCA1 and/or BRCA2. Women with both $B R C A 1$ and $B R C A 2$ mutations were included in downstream analyses. Data were available for year of birth, age at study recruitment, age at cancer diagnosis, $B R C A 1$ and $B R C A 2$ mutation description and self-reported ethnicity. Women with ovarian cancer history were not excluded from analyses, and they represented $15 \%$ and $7 \%$ of $B R C A 1$ and BRCA2 mutation carriers, respectively. Information regarding mastectomy was incomplete and was therefore not used as an inclusion or exclusion parameter.

\section{Genotyping and quality filtering}

Genotyping was conducted using the iCOGS custom Illumina Infinium array. Data from this array are available to the scientific community upon request. Please see [37] for more information. Genotypes were called using Illumina's proprietary GenCall algorithm. Genotyping and quality filtering were described previously $[35,36]$. Initially, 129 mitochondrial SNPs were genotyped for both $B R C A 1$ and BRCA2 mutation carriers. SNPs fulfilling the following criteria were excluded from downstream analyses: monoallelic SNPs (minor allele frequency $=0$ ), SNPs with more than $5 \%$ data missing, annotated as triallelic, or having probes cross-matching with the nuclear genome. Heterozygous genotypes were removed from analyses, and we further filtered out SNPs having more than $5 \%$ of heterozygous calls to limit the potential for heteroplasmy affecting our results. We also did not retain SNPs representing private mutations. These mutations are rare, often restricted to a few families, and not sufficiently prevalent in the general population to be included in the reference 
mitochondrial evolutionary tree (see below). This last step of filtration yielded 93 and 92 SNPs for the pop1 and pop 2 analyses, respectively (see Additional file 2). Only individuals with fully defined haplotypes (that is, nonmissing genotypes for the 93 and 92 SNPs selected for pop 1 and pop 2, respectively) were included in downstream analyses (14,536 and 7,678 individuals, respectively).

\section{Mitochondrial genome evolution and haplogroup definition}

Analyses were based on the theoretical reconstructed phylogenetic tree of the mitochondrial genome (mtTree) known as PhyloTree [38] (v.15). The mtTree is rooted by the Reconstructed Sapiens Reference Sequence (RSRS).
RSRS has been identified as the most likely candidate to root the mtTree by refining human mitochondrial phylogeny by parsimony [39]. Each haplogroup in mtTree is defined by the set of mtDNA SNPs that have segregated in RSRS until today in the mitochondrial genome. Each haplogroup is fully characterized by the 16,569-bp sequence resulting from the application of all the substitutions that are encoded by the corresponding SNPs in the RSRS sequence.

\section{Haplogroups imputation}

The phylogenetic approach used to infer haplogroups is described in Figure 1. Mitochondrial genome sequences can be reconstructed at each node of mtTree, given the

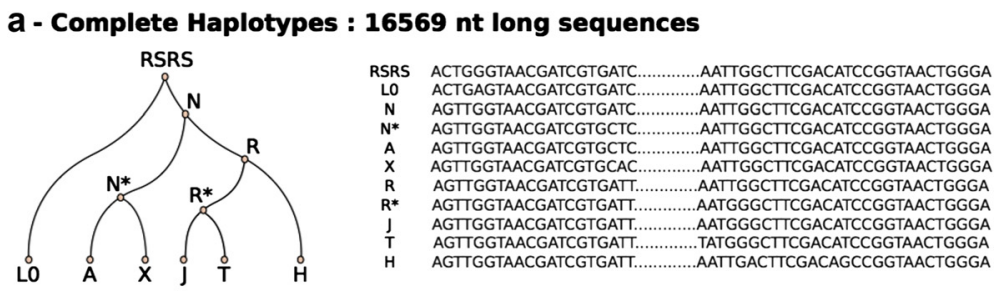

b- Short Haplotypes : 92 nt long sequences

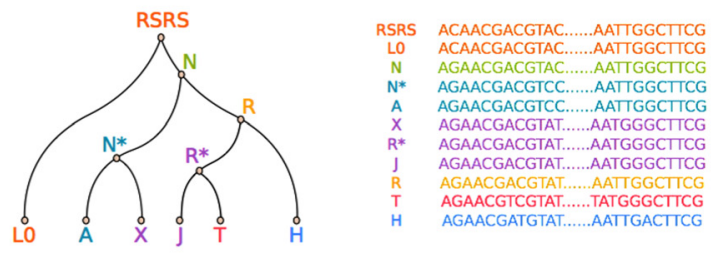

C - When a short haplotype corresponds to only one haplogroup

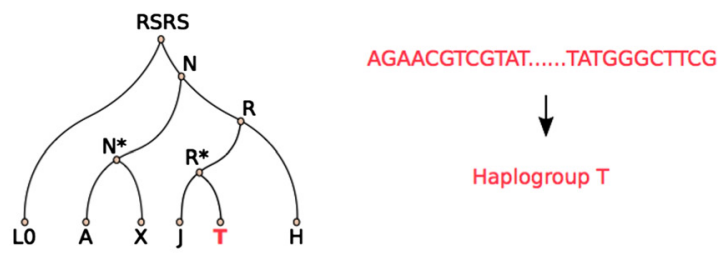

d - When a short haplotype corresponds to several haplogroups

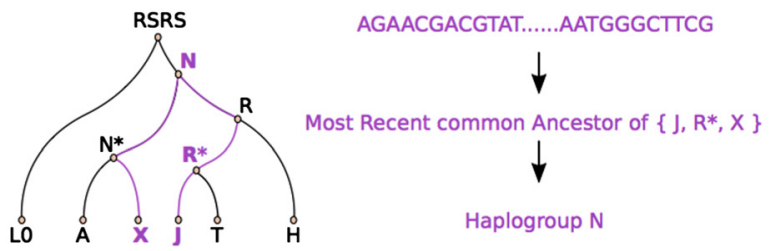

Figure 1 Simplified representation of the phylogenic method used to infer haplogroups. (a) Full-length haplotypic sequences are reconstructed at each node of the reference tree. (b) Haplotypes are then restricted to available loci. Sequences of the same color are identical. (c) Unique short haplotypes are matched directly with the corresponding haplogroup. (d) Sequences that match with several haplogroups are associated with their most recent common ancestor haplogroup. RSRS, Reconstructed Sapiens Reference Sequence. 
substitutions that have segregated in RSRS. Each haplogroup therefore has a corresponding full-length mitochondrial sequence. However, the full-length mitochondrial sequence is not available in the data, because the iCOGS platform captured only 93 and 92 SNPs for pop 1 and pop2, respectively. Thus, for each of the 7,864 nodes of the phylogenetic tree, the corresponding short haplotype (that is, the full-length sequence restricted to available loci) was defined. Some of the short haplotypes are unique, and they can be matched with their corresponding haplogroup directly. However, most of the time, given the small number of SNPs analyzed, several haplogroups correspond to the same short haplotype. Consequently, a unique haplogroup cannot confidently be assigned to each short haplotype. Therefore, each short haplotype was assigned the most recent common ancestor of all the haplogroups that share the same short haplotype. Once this matching was done, short haplotypes were reconstructed in the same way for each individual in our dataset and were assigned the corresponding haplogroup. The accuracy of the method used was assessed by application to a set of $630 \mathrm{mtDNA}$ sequences of known European and Caucasian haplogroups (see Additional file 3).

\section{Association detection}

This phylogenetic approach is based on the identification of subclades in the reference phylogenetic tree of the mitochondrial genome differentially enriched for cases and unaffected controls compared with neighboring subclades. We used ALTree [33,34] to perform association testing. ALTree-standing for Association detection and Localization of susceptibility sites using haplotype phylogenetic Trees-is an algorithm used to perform nested homogeneity tests to compare distributions of affected and unaffected individuals in the different clades of a given phylogenetic tree. The objective is to detect if some clades of a phylogenetic tree are more or less enriched in affected or unaffected individuals compared with the rest of the tree. There are as many tests performed as there are levels in the phylogenetic tree. The $P$-value at each level of the tree is obtained by a permutation procedure in which 1,000 permutations are performed. Individual labels ("affected" or "unaffected") are permutated 1,000 times to see to what extent the observed distribution of affected or unaffected is different from a random distribution. A procedure to correct for multiple testing adapted to nested tests [40] is implemented in ALTree. The objective of ALTree is to detect an enrichment difference at the level of the whole tree. To conserve computational time and resources, only the most significant $P$-value obtained for all tests performed on one tree is corrected.

\section{Handling genetic dependency}

ALTree is used to perform homogeneity tests to detect differences in enrichment or depletion of affected or unaffected individuals between clades in the phylogenetic tree. This kind of test can be performed only on independent data. However, because some individuals in the CIMBA dataset belong to the same family, we constructed datasets with genetically independent data by randomly selecting one individual from among all those belonging to the same family and sharing the same short haplotype. To take into account the full variability of our data, we resampled 1,000 times. The results of the analysis pipeline are obtained for each resampling independently and then averaged over the 1,000 resamplings to obtain final results.

\section{Character reconstruction at ancestral nodes}

Before the ALTree localization algorithm was launched, ancestral sequences were reconstructed at each internal tree node; that is, short haplotypes were inferred with maximum likelihood at all nodes that were not leaves. We used the software PAML [41] to perform the reconstruction at ancestral nodes using a maximum likelihood method. The phylogeny model used was the general time-reversible model (either GTR or REV).

\section{Localization of susceptibility sites}

ALTree also includes an algorithm used to identify which sites are the most likely ones to be involved in the association detected. For each short haplotype observed, the ALTree add-on altree-add-S adds to the short haplotype sequence a supplementary character called $S$, which represents the disease status associated with this short haplotype. Are individuals carrying this short haplotype more often affected or unaffected? $S$ is calculated based on the affected and unaffected counts, the relative proportion of affected and unaffected in the whole dataset, and sensibility parameter $\varepsilon$. $\varepsilon$ was set to its default value, which is 1 . After $S$ character computation, haplotypes including character $S$ are reconstructed at ancestral nodes. Susceptibility site localization is achieved with ALTree by computing a correlated evolution index calculated between each change of each site and the changes of the character $S$ in the two possible directions of change. The sites whose evolution are the most correlated with the character $S$ are the most likely susceptibility sites.

\section{Selected subclades}

The analyses were carried out on the full evolutionary tree. However, the more haplogroups there are at each level, the less statistical power homogeneity tests have. Therefore, analyses were also applied to subclades extracted from the tree. Subclades were defined using counts of individuals in each haplogroup of the clade to maximize statistical power. The chosen subclades 
and corresponding affected and unaffected counts are presented in Table 2.

\section{Statistical analysis}

We quantified the effect associated with enrichment discovered by applying ALTree by building a weighted Cox regression in which the outcome variable is the status (affected or non-affected) and the explicative variable is the inferred haplogroup. Analyses were stratified by country. Data were restricted to the clades of interest. The uncertainty in haplogroup inference was not taken into account in the model. The weighting method used takes into account breast cancer incidence rate as a function of age [42] and the gene containing the observed pathogenic mutation (that is, BRCA1 or $B R C A 2)$. Familial dependency was handled by using a robust sandwich estimate of variance ( $\mathrm{R}$ package survival, cluster() function).

\section{Results}

\section{Haplogroup imputation}

In Additional file 4, absolute and relative frequencies are recapitulated for each haplogroup imputed in $B R C A 1$ and BRCA2 mutation carriers. For BRCA1 mutation carriers, we reconstructed 489 distinct short haplotypes of 93 loci from the genotypes data. Only 162 of those 489 short haplotypes matched theoretical haplotypes reconstructed in the reference mitochondrial evolutionary tree. These 162 haplotypes represented 13,315 of 14,536 individuals. Thus, $91.6 \%$ of $B R C A 1$ mutation carriers were successfully assigned a haplogroup. For BRCA2 mutation carriers, we reconstructed 350 distinct short haplotypes of 92 loci from our genotype data. Only 139 of those 350 short haplotypes matched theoretical haplotypes reconstructed in the reference mitochondrial evolutionary tree. These 139 haplotypes represented 6,996 of 7,678 individuals. Thus, $91.1 \%$ of $B R C A 2$ mutation carriers were successfully assigned a haplogroup. Because more $B R C A 1$ than $B R C A 2$ mutation carriers were genotyped (14,536 vs. 7,678 individuals), we logically observed more distinct haplotypes in pop1 than in pop2 (489 vs. 350 haplotypes).

The accuracy of the main haplogroup inference method used was estimated at $82 \%$ and reached $100 \%$ for haplogroups I, J, K, T, U, W and X. Given the set of SNPs we disposed of, our method has difficulty differentiating between $\mathrm{H}$ and $\mathrm{V}$ haplogroups (see Additional file 3).

\section{Association results}

For both populations of $B R C A 1$ or BRCA2 mutation carriers, as well as for the full tree as for all selected subclades (see Table 1), we extracted the mean corrected $P$-values for association testing over all resamplings performed (see Table 2 ). The only corrected $P$-value that remained significant was that obtained for subclade $T$
Table 1 Counts of participants in selected subclades

\begin{tabular}{lll}
\hline Subclade & BRCA1 mutation carriers & BRCA2 mutation carriers \\
\hline U8 & 1,458 & 863 \\
T & 1,243 & 651 \\
$\mathrm{~J}$ & 1,270 & 630 \\
$\mathrm{~J} 1$ & 1,043 & 513 \\
H & 3,706 & 1,967 \\
H1 & 582 & 337 \\
U5 & 868 & 458 \\
X1'2'3 & 221 & 103 \\
K1a & 608 & 364 \\
\hline
\end{tabular}

(abbreviated $\mathrm{T}^{*}$ ) in the population of individuals of BRCA2 mutation carriers $(P=0.04)$.

The phylogenetic tree of subclade $\mathrm{T}$ (see Figure 2a) contains only three levels; thus, only three tests were performed within this clade. Raw $P$-values were examined to determine at which level of the tree ALTree detects a difference of enrichment in affected or unaffected individuals (see Table 3 ). Only the $P$-value associated with the test performed at the first level of the tree is significant. We looked more closely at the mean frequencies of affected and unaffected individuals in the tree at this level (see Figure $2 \mathrm{~b}$ ). In the T1a1 subclade, the mean count of affected and unaffected are 32 and 47, respectively. In the T2\% subclade, we observed, on average, 217 and 148 affected and unaffected individuals, respectively, whereas in the $\mathrm{T}$ subclade, we observed, on average, 13 and 11 affected and unaffected individuals, respectively. The ranges observed for each of these values over the 1,000 resamplings are represented in Figure 2b. On the basis of these observations, we conclude that subclade T1a1 is depleted in affected carriers compared with the neighboring subclades $\mathrm{T}$ and $\mathrm{T} 2$.

$\begin{aligned} & \text { Table } 2 \text { Mean corrected } \boldsymbol{P} \text {-values for association testing } \\
& \text { with ALTree }\end{aligned}$
\begin{tabular}{lll}
\hline Subclade & pop1 corrected $\boldsymbol{P}$-value & pop2 corrected $\boldsymbol{P}$-value \\
\hline Full & 0.830 & 0.681 \\
U8 & 0.146 & 0.626 \\
T & 0.285 & $\mathbf{0 . 0 4 0}$ \\
J & 0.718 & 0.112 \\
J1 & 0.621 & 0.150 \\
H & 0.747 & 0.930 \\
H1 & 0.268 & 0.804 \\
U5 & 0.829 & 0.747 \\
X1'2 '3 & 0.416 & 0.629 \\
K1a & 0.170 & 0.162 \\
\hline
\end{tabular}

apop1, BRCA1 mutation carrier; pop2, BRCA2 mutation carrier. Bold indicates a significant $P$-value. 
a

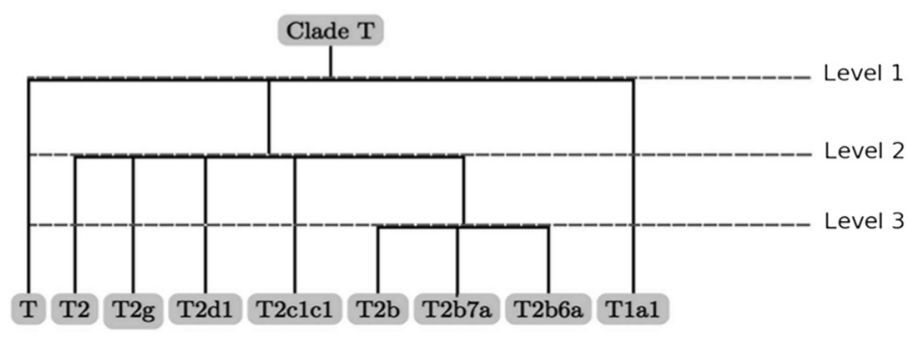

b

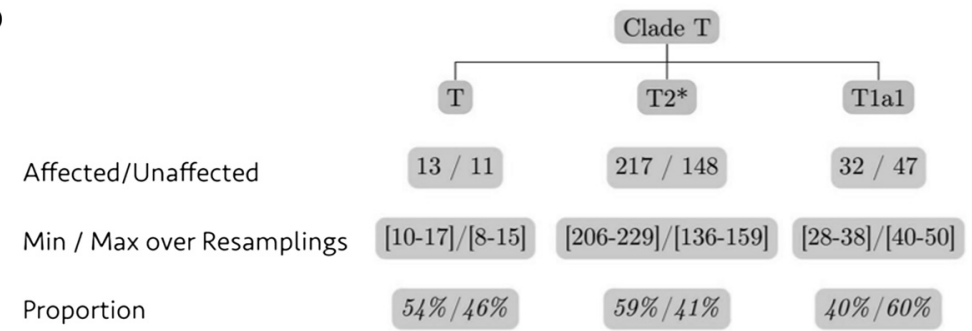

Figure 2 Phylogenetic tree of subclade T tested for association with ALTree. (a) Phylogenetic tree of subclade T with all observed haplogroups. A homogeneity test is performed at each level of the tree. (b) First level of the phylogenetic tree of subclade T. Averaged counts, ranges and proportions of affected and unaffected observed in resamplings are indicated below each subclade. T2* represents the entire T2 subclade.

\section{Localization results}

We performed a localization analysis with ALTree. The correlated evolution index for all non-monomorphic sites observed in short haplotype sequences of subclade $\mathrm{T}$ are displayed in Additional file 5. The higher the correlated evolution index, the more likely it is that corresponding sites will be involved in the observed association. Three short haplotype sites numbered 44, 57 and 72 and corresponding to SNPs T988C, G11812A/rs4154217 and G13708A/rs28359178, respectively, clearly distinguish themselves, with correlation index values of $0.390,0.324$ and 0.318 , respectively, whereas the correlation index values of all other sites ranged from -0.270 to -0.101 . Table 4 shows the details for these three loci.

\section{Effect quantification}

The ALTree method is able to detect an association, but cannot to quantify the associated effect. We estimated the risk of breast cancer for individuals with the T1a1 haplogroup compared with individuals with another $\mathrm{T}$ subclade haplogroup in the population of BRCA2 mutation carriers using a more classical statistical method, a weighted Cox regression. We found a breast cancer HR of 0.55 (95\% CI, 0.34 to $0.88 ; P=0.014$ ). We also tested haplogroup

Table 3 Non-corrected $\boldsymbol{P}$-values by level of phylogenetic tree for subclade $T$ in $B R C A 2$ mutation carriers

\begin{tabular}{lll}
\hline Level & Degrees of freedom & Mean of non-corrected $P$-value \\
\hline 1 & 2 & 0.02141039 \\
2 & 6 & 0.14355900 \\
3 & 8 & 0.22249700 \\
\hline
\end{tabular}

T1a1 and compared it with other $\mathrm{T}^{*}$ haplogroups and the $\mathrm{H}$ haplogroup (the main haplogroup in the general population), and we found a breast cancer HR of 0.62 ( $95 \%$ CI, 0.40 to $0.95 ; P=0.03$ ).

\section{Discussion}

We employed an original phylogenetic analytic method, coupled with more classical molecular epidemiologic analyses, to detect mitochondrial haplogroups differentially enriched for affected $B R C A 1 / 2$ mutation carriers. We successfully inferred haplogroups for more than $90 \%$ of individuals in our dataset. After haplogroup imputation, the ALTree method identified T1a1 in the T clade as differentially enriched in affected $B R C A 2$ mutation carriers, whereas no enrichment difference was found for BRCA1 mutation carriers. The $\mathrm{T}$ subclade is present in $4 \%$ of African populations compared with $11 \%$ in Caucasian and Eastern European populations [43]. In our data, the $\mathrm{T}$ subclade represented $9.34 \%$ of $B R C A 1$ mutation carriers and $9.30 \%$ of BRCA2 carriers. The ALTree method also identified three potential breast cancer susceptibility loci in mtDNA. The main goals of using the phylogenetic method we used were to improve statistical power by regrouping subclades according to genetic considerations, to limit the number of tests performed and to precisely quantify this number. ALTree identified three SNPs of interest. Whereas the association we observed could possibly be driven by a single SNP, no difference was observed between multivariate and univariate cox models including the three SNPs identified by ALTree (data not shown).

In this study, we investigated to what extent mtDNA variability modified breast cancer risk in individuals 
Table 4 Description of loci identified as potential susceptibility sites by ALTree ${ }^{a}$

\begin{tabular}{llllllll}
\hline Site & SNP name & Position & Direction of change & Correlated evolution index & Major allele & Minor allele & MAF in pop2 \\
\hline 44 & MitoT9900C & 9,899 & $\mathrm{~T} \rightarrow \mathrm{C}$ & 0.390 & $\mathrm{~T}$ & $\mathrm{C}$ & 0.016 \\
57 & rs41544217 & 11,812 & $\mathrm{G} \rightarrow \mathrm{A}$ & 0.324 & $\mathrm{~A}$ & $\mathrm{G}$ & 0.071 \\
72 & rs28359178 & 13,708 & $\mathrm{G} \rightarrow \mathrm{A}$ & 0.318 & $\mathrm{G}$ & $\mathrm{A}$ & 0.111 \\
\hline
\end{tabular}

${ }^{\mathrm{a}} \mathrm{MAF}$, Mean allele frequency; pop2, BRCA2 mutation carrier.

carrying pathogenic mutations in $B R C A 1 / 2$. A large proportion of breast cancer heritability still remains unexplained today [44]. Different methods exist to study genomic susceptibility to a disease, such as linkage analyses (which identified the BRCA1 and BRCA2 susceptibility genes) or genome-wide association studies (GWASs). However, classical linkage analysis cannot be applied to the haploid mitochondrial genome. Furthermore, commercial GWAS chips available do not adequately capture the majority of mtDNA SNPs. A non-genome-wide and mtDNA-focused approach was required to explore how mtDNA variability influences breast cancer risk. Here we have shown that BRCA2 mutation carriers with the subclade T1a1 have between $30 \%$ and $50 \%$ less risk of breast cancer than those with other clades, which, if validated, is a clinically meaningful risk reduction and may influence the choice of risk management strategies.

The association we observed among BRCA2, but not $B R C A 1$, mutation carriers may reveal a functional alteration that would be specific to mechanisms involving $B R C A 2$-related breast cancer. Today, it is established that $B R C A 1$ - and BRCA2-associated breast cancers are not phenotypically identical. These two types of tumors do not harbor the same gene expression profiles or copy number alterations [45]. Breast cancer risk modifiers in BRCA1/2 mutation carriers have already been identified [46]. However, most of them are specific from one or the other type of mutation carried [47]. It is therefore not surprising that this observation is observed in BRCA2 mutation carriers only.

Our inability to assign haplogroups to $9 \%$ of study participants could have three main explanations. First, given the high mutation rate in the mitochondrial genome, observed combinations of mtDNA SNPs might have appeared relatively recently in the general population, and the corresponding haplotypes might not yet be incorporated into PhyloTree. Second, only one genotyping error could lead to chimeric haplotypes that do not exist, although, given the quality of our genotyping data, this is unlikely. Third, the mitochondrial reference evolutionary tree PhyloTree is based on phylogeny reconstruction by parsimony, and, for some subclades, it might be suboptimal, especially for haplogroups relying on few mitochondrial sequences, as is the case for African haplogroups [48]. In cases of uncertainty, the choice we made to assign the most recent common ancestor to the studied haplotype enabled us to improve statistical power without introducing a bias in the detected association. For the association detected between $\mathrm{T}, \mathrm{T} 1 *$ and $\mathrm{T} 2 *$ subclades, the haplogroup inference method used did not bias the counts of affected and unaffected individuals in these subclades. More details are presented in Additional file 6. Furthermore, on the basis of the haplogroup inference with our method of 630 European and Caucasian mtDNA sequences whose haplogroup is known, we successfully assigned the correct main haplogroup and subhaplogroup of $100 \%$ of sequences belonging to $\mathrm{T}, \mathrm{T} 2 *$ and $\mathrm{T} 1 \mathrm{a} 1 *$ haplogroups.

We quantified the effect corresponding to the detected association by using a more classical approach. We built a weighted Cox regression including inferred haplogroup as an explicative variable. However, the uncertainty in haplogroup inference was not taken into account in this model. Nevertheless, based on haplogroup assignment and regrouping performed in clade $\mathrm{T}$, affected and unaffected counts of individuals in this clade were not biased.

With only 129 loci genotyped over the 16,569 nucleotides composing the mitochondrial genome, we certainly did not explore the full variability of mitochondrial haplotypes. A characterization of individual mitochondrial genomes would require more complete data acquisition methods to be used, such as next-generation sequencing. However, next-generation sequencing has its own limits and challenges, because some regions of the mitochondrial genome are not easily mappable, owing to a high homology with the nuclear genome, among other factors, and important bioinformatics treatment is necessary to overcome sequencing technology biases. Finally, even for a relatively short genome of "only" $16,569 \mathrm{bp}$, mtDNA sequencing of more than 20,000 individuals would represent a major increase in cost relative to genotyping 129 SNPs.

ALTree identified T9899C, G11812A/rs41544217 and G13708A/rs28359178 as three potential susceptibility sites for the discovered association (see Additional file 7). These three SNPs are located in the coding part of genes MT-CO3, MT-ND4 and MT-ND5, respectively. When looking at PhyloTree, T9899C seems to be involved in T1 subclade definition, whereas G13708A and A11812G are involved in T2 subclade definition. Whereas T98899C and 
G11821/rs41544217 are synonymous SNPs, G10398A leads to a change of amino acid in the final protein (from alanine to threonine). These two synonymous SNPs have never been described in a disease context in the literature. G13708A is also known for being a secondary mutation for Leber's hereditary optic neuropathy (LHON) and multiple sclerosis [49]. Although the role of secondary mutations in LHON is still controversial, G13708A could be associated with impairment of the respiratory chain in this pathology. G13708A has also been described as a somatic mutation in a breast cancer tumor, whereas it was not present in adjacent normal tissue or in blood leukocytes [50]. A high proportion of mitochondrial somatic tumor-specific variants are also known mtDNA SNPs, which is consistent with the hypothesis that tumor cells are prone to acquire the same mutations that segregate into mtDNA by selective adaptation when humans migrated out of Africa and confronted new environments [51]. Interestingly, the germline variant G13708A has already been shown to be inversely associated with familial breast cancer risk (with the same direction of the association), with a breast cancer odds ratio of 0.47 ( $95 \% \mathrm{CI}, 0.24$ to 0.92) [52]. None of these SNPs have been described in the context of ovarian cancer.

The corrected $P$-value obtained using ALTree in studying clade $\mathrm{T}$ is 0.02 , which is not highly significant. A replication step should be performed to validate these results. However, it will be difficult to include enough women in this replication step, given the specific profile studied here. In fact, the estimations of BRCA2 pathogenic mutations in the general population range from $0.068 \%$ [5] to $0.69 \%$ [53]. T1a1 represents only a small percentage of European haplogroups (from 1\% to 2\%). The number of women who have this association is therefore low. However, women carrying such mutations are confronted with drastic choices regarding the prevention of breast cancer, notably prophylactic mastectomy or complete hysterectomy. If breast cancer risk is really reduced by a factor of 2 for women with T1a1, this could be an important fact to take into account for breast cancer prevention.

\section{Conclusions}

This study and our results suggest that mitochondrial haplogroup T1a1 may modify the individual breast cancer risk in BRCA2 mutation carriers. For now, this observation cannot be extended to the general population. Further investigation of the biological mechanism behind the associations we observed may further reinforce the hypothesis that the mitochondrial genome is influential in breast cancer risk, particularly among carriers of $B R C A 2$ mutations, and, if validated, is of a level to influence cancer risk management choices.

\section{Additional files} Additional file 1: List of ethical committees that approved the
access to the data analyzed in this study.

Additional file 2: SNPs selected for downstream analyses.

Additional file 3: Description and results of the procedure used to estimate the accuracy of our haplogroup inference methodology.

Additional file 4: Absolute and relative frequencies of imputed haplogroups by population. Table containing absolute and relative frequencies of imputed haplogroups for BRCA1 and BRCA2 mutation carriers.

Additional file 5: Correlated evolution index for all non-monomorphic sites observed in short haplotype sequences of subclade T. Table containing correlated evolution index for all non-monomorphic sites observed in short haplotypes sequences of subclade $\mathrm{T}$.

Additional file 6: Details of haplogroups inference results for subclade $T$.

Additional file 7: Methods used to compute coevolution index.

\section{Abbreviations}

BCAC: Breast Cancer Association Consortium; Cl: Confidence interval; CIMBA: Consortium of Investigators of Modifiers of BRCA1/2;

COGS: Collaborative Oncological Gene-environment Study; DSB: Doublestrand break; GWAS: Genome-wide association study; HR: Hazard ratio; LHON: Leber's hereditary optic neuropathy; MAF: Mean allele frequency; mtDNA: Mitochondrial DNA; mtTree: Phylogenetic tree of the mitochondrial genome; OCAC: Ovarian Cancer Association Consortium; pop1: BRCA1 mutation carrier; pop2: BRCA2 mutation carrier; PRACTICAL: Prostate Cancer Association Group to Investigate Cancer Associated Alterations in the Genome; ROS: Reactive oxygen species; RSRS: Reconstructed Sapiens Reference Sequence; SNP: Single-nucleotide polymorphism.

\section{Competing interests}

The authors declare that they have no competing interests.

\section{Authors' contributions}

Conception and design: DGC and GT. Development of methodology: SB, CBa and VD. Acquisition of data: LM, SHe, DB, ALe, JD, KBK, PS, MBT, WKC, DEG, SSB, RJ, LT, NT, CMD, EJVR, SLN, YCD, AMG, BE, FCN, TVOH, AO, JBe, RA, ES, JNW, MThe, PP, PR, VP, RDO, BB, BP, DZ, GSC, SMan, LV, GLC, LP, LO, DY, IK, JGa, UH, AD, ABr, CBr, CF, DGE, DF, DE, FDo, JCo, JA, JBa, LW, LI, LES, MJK, MTi, MTR, MEP, PJM, RP, RE, RDa, SHo, TCo, AKG, Cl, KC, KDL, AM, AG, BW, CS, CE, DN, DS, HP, KK, KR, ND, NA, RV, RKS, SP, NB, SW, AdP, CLe, CLas, DL, ER, FDa, GSC, HD, LB, LG, NU, VB, VS, YB, JCa, LVL, MP, PAD, MdlH, TCa, HN, KA, AJag, AMvdO, CMK, CMA, FEvL, FBH, HEM, JCO, KvR, MAR, PD, RBvdL, EO, OD, AT, CLaz, IB, JDV, AJak, GSu, JGr, JLu, KD, KJ, BAA, CM, AA, MM, MRT, ABS, WF, CO, NLi, VSP, CIS, ALinc, LJ, MC, MR, JV, ABe, AF, CFS, CR, DGK, GP, MTe, MHG, PLM, GR, El, AMM, GG, ILA, ST, AET, ISP, MTho, TAK, UBJ, MAC, EF, JZ, YL, ALind, BM, BA, NLo, RR, OIO, RLN, SR, KLN, SMD, TRR, BKA, GM, BYK, JLe, SO, DSL, GT, JS, FJC, KO, DFE, GC, ACA, SMaz, CMP and OMS. Analysis and interpretation of data: SB, DGC and ACA. Writing of the manuscript: SB, DGC, ACA, SH, ABS, GC, SLN, AET, ILA, JCO, KO, MTho and GM. All authors read and approved the final manuscript.

\section{Acknowledgements}

Collaborative Oncological Gene-environment Study (COGS): This study would not have been possible without the contributions of the following: Per Hall (COGS); Kyriaki Michailidou, Manjeet K Bolla and Qin Wang (Breast Cancer Association Consortium (BCAC)); Rosalind A Eeles, Ali Amin Al Olama, Zsofia Kote-Jarai and Sara Benlloch (PRACTICAL); Alison M Dunning, Craig Luccarini, Michael Lush and the staff of the Centre for Cancer Genetic Epidemiology; Simard and Daniel C Tessier, Francois Bacot, Daniel Vincent, Sylvie LaBoissière and Frederic Robidoux and the staff of the McGill University and Génome Québec Innovation Centre; and Julie M Cunningham, Sharon A Windebank, Christopher A Hilker, Jeffrey Meyer and the staff of Mayo Clinic Genotyping Core Facility.

Consortium of Investigators of Modifiers of BRCA1/2 (CIMBA): Maggie Angelakos, Judi Maskiell, Gillian Dite and Helen Tsimiklis; members of and participants in the New York site of the Breast Cancer Family Registry; 
members of and participants in the Ontario Familial Breast Cancer Registry for their contributions to the study; Vilius Rudaitis, Laimonas Griškevičius, Drs Janis Eglitis, Anna Krilova and Aivars Stengrevics; the families who contribute to the BRCA-gene mutations and breast cancer in South African women (BMBSA) study; Chun Ding and Linda Steele; Alicia Barroso, Rosario Alonso, Guillermo Pita, Alessandra Viel and Lara della Puppa of the Centro di Riferimento Oncologico, IRCCS, Aviano (PN), Italy; Laura Papi of the University of Florence, Florence, Italy; Monica Barile of the Istituto Europeo di Oncologia, Milan, Italy; Liliana Varesco of the IRCCS AOU San Martino - IST Istituto Nazionale per la Ricerca sul Cancro, Genoa, Italy; Stefania Tommasi, Brunella Pilato and Rossana Lambo of the Istituto Nazionale Tumori "Giovanni Paolo II" - Bari, Italy; Aline Martayan of the Istituto Nazionale Tumori Regina Elena, Rome, Italy; Maria Grazia Tibiletti of the Ospedale di Circolo-Università dell'Insubria, Varese, Italy; and the personnel of the Cogentech Cancer Genetic Test Laboratory, Milan, Italy.

Epidemiological Study of BRCA1 and BRCA2 Mutation Carriers (EMBRACE) Collaborating Centers: coordinating center, Cambridge, UK: Debra Frost, Steve Ellis, Elena Fineberg and Radka Platte; North of Scotland Regional Genetics Service, Aberdeen, UK: Zosia Miedzybrodzka and Helen Gregory; Northern Ireland Regional Genetics Service, Belfast, UK: Patrick Morrison and Lisa Jeffers; West Midlands Regional Clinical Genetics Service, Birmingham, UK: Trevor Cole, Kai-ren Ong and Jonathan Hoffman; South West Regional Genetics Service, Bristol, UK: Alan Donaldson and Margaret James; East Anglian Regional Genetics Service, Cambridge, UK: Marc Tischkowitz, Joan Paterson and Amy Taylor; Medical Genetics Services for Wales, Cardiff, UK: Alexandra Murray, Mark T Rogers and Emma McCann; St James's Hospital, Dublin, and National Centre for Medical Genetics, Dublin, Ireland: M John Kennedy and David Barton; South East of Scotland Regional Genetics Service, Edinburgh, UK: Mary Porteous and Sarah Drummond; Peninsula Clinical Genetics Service, Exeter, UK: Carole Brewer, Emma Kivuva, Anne Searle, Selina Goodman and Kathryn Hill; West of Scotland Regional Genetics Service, Glasgow, UK: Rosemarie Davidson, Victoria Murday, Nicola Bradshaw, Lesley Snadden, Mark Longmuir, Catherine Watt, Sarah Gibson, Eshika Haque, Ed Tobias and Alexis Duncan; South East Thames Regional Genetics Service, Guy's Hospital London: Louise Izatt, Chris Jacobs and Caroline Langman; North West Thames Regional Genetics Service, Harrow, UK: Huw Dorkins; Leicestershire Clinical Genetics Service, Leicester, UK: Julian Barwell; Yorkshire Regional Genetics Service, Leeds, UK: Julian Adlard and Gemma Serra-Feliu; Cheshire \& Merseyside Clinical Genetics Service, Liverpool, UK: Ian Ellis and Catherine Houghton; Manchester Regional Genetics Service, Manchester, UK: D Gareth Evans, Fiona Lalloo and Jane Taylor. North East Thames Regional Genetics Service, NE Thames, London: Lucy Side, Alison Male and Cheryl Berlin; Nottingham Centre for Medical Genetics, Nottingham, UK: Jacqueline Eason and Rebecca Collier; Northern Clinical Genetics Service, Newcastle, UK: Fiona Douglas, Oonagh Claber and Irene Jobson; Oxford Regional Genetics Service, Oxford, UK: Lisa Walker, Diane McLeod, Dorothy Halliday, Sarah Durell and Barbara Stayner; The Institute of Cancer Research and Royal Marsden NHS Foundation Trust, London: Ros Eeles, Susan Shanley, Nazneen Rahman, Richard Houlston, Elizabeth Bancroft, Elizabeth Page, Audrey Ardern-Jones, Kelly Kohut, Jennifer Wiggins, Elena Castro, Emma Killick, Sue Martin, Gillian Rea and Anjana Kulkarni; North Trent Clinical Genetics Service, Sheffield, UK: Jackie Cook, Oliver Quarrell and Cathryn Bardsley; South West Thames Regional Genetics Service, London: Shirley Hodgson, Sheila Goff, Glen Brice, Lizzie Winchester, Charlotte Eddy, Vishakha Tripathi, Virginia Attard and Anna Lehmann; Wessex Clinical Genetics Service, Princess Anne Hospital, Southampton, UK: Diana Eccles, Anneke Lucassen, Gillian Crawford, Donna McBride and Sarah Smalley; and JoEllen Weaver and Dr Betsy Bove for their technical support. Genetic Modifiers of Cancer Risk in BRCA1/2 Mutation Carriers (GEMO) study: National Cancer Genetics Network UNICANCER Genetic Group, France; GEMO Collaborating Centers: coordinating centers, Unité Mixte de Génétique Constitutionnelle des Cancers Fréquents, Hospices Civils de Lyon - Centre Léon Bérard, and equipe Génétique du cancer du sein, Centre de Recherche en Cancérologie de Lyon: Olga Sinilnikova, Sylvie Mazoyer, Francesca Damiola, Laure Barjhoux, Carole Verny-Pierre, Alain Calender, Sophie Giraud and Mélanie Léone; and Service de Génétique Oncologique, Institut Curie, Paris: Dominique Stoppa-Lyonnet, Marion Gauthier-Villars, Bruno Buecher, Claude Houdayer, Virginie Moncoutier, Muriel Belotti, Carole Tirapo, Antoine de Pauw. Institut Gustave Roussy, Villejuif, France: Brigitte Bressac-de-Paillerets and Olivier Caron; Centre Jean Perrin, Clermont-Ferrand, France: Yves-Jean Bignon and Nancy Uhrhammer; Centre Léon Bérard, Lyon, France: Christine Lasset, Valérie Bonadona and Sandrine Handallou; Centre François Baclesse, Caen,
France: Agnès Hardouin and Pascaline Berthet; Institut Paoli Calmettes, Marseille, France: Hagay Sobol, Violaine Bourdon, Tetsuro Noguchi, Audrey Remenieras and François Eisinger; Centre Hospitalier Régional Universitaire Arnaud-de-Villeneuve, Montpellier, France: Isabelle Coupier and Pascal Pujol; Centre Oscar Lambret, Lille, France: Jean-Philippe Peyrat, Joëlle Fournier, Françoise Révillion, Philippe Vennin and Claude Adenis; Hôpital René Huguenin, Institut Curie, Saint-Cloud, France: Etienne Rouleau, Rosette Lidereau, Liliane Demange and Catherine Nogues; Centre Paul Strauss, Strasbourg, France: Danièle Muller and Jean-Pierre Fricker; Institut Bergonié, Bordeaux, France: Emmanuelle Barouk-Simonet, Françoise Bonnet, Virginie Bubien, Nicolas Sevenet and Michel Longy; Institut Claudius Regaud, Toulouse, France: Christine Toulas, Rosine Guimbaud, Laurence Gladieff and Viviane Feillel; Centre Hospitalier Universitaire de Grenoble, Grenoble, France: Dominique Leroux, Hélène Dreyfus, Christine Rebischung and Magalie Peysselon; Centre Hospitalier Universitaire de Dijon, Dijon, France: Fanny Coron and Laurence Faivre; Centre Hospitalier Universitaire de Saint-Etienne, Saint-Etienne, France: Fabienne Prieur, Marine Lebrun and Caroline Kientz. Hôtel Dieu Centre Hospitalier, Chambéry, France: Sandra Fert Ferrer; Centre Antoine Lacassagne, Nice, France: Marc Frénay; Centre Hospitalier Universitaire de Limoges, Limoges, France: Laurence Vénat-Bouvet; Centre Hospitalier Universitaire de Nantes, Nantes, France: Capucine Delnatte; Centre Hospitalier Universitaire de Bretonneau, Tours, France: Isabelle Mortemousque; Groupe Hospitalier Pitié-Salpétrière, Paris: Florence Coulet, Chrystelle Colas and Florent Soubrier; Centre Hospitalier Régional Universitaire de Nancy, Vandœuvre-lès-Nancy, France: Johanna Sokolowska and Myriam Bronner; Hôpital Jean Minjoz, Centre Hospitalier Universitaire de Besançon, France: Marie-Agnès Collonge-Rame and Alexandre Damette; Creighton University, Omaha, NE, USA: Henry T Lynch and Carrie L Snyder; the technical support of Ilse Coene en Brecht Crombez; and the investigators of the Australia New Zealand Gynaecological Oncology Group (ANZGOG). We acknowledge Alicia Tosar for her technical assistance; Taru A Muranen, Drs Carl Blomqvist and Kirsimari Aaltonen, and registered nurses Irja Erkkilä and Virpi Palola for their help with the Helsinki Breast Cancer Study (HEBCS) data and samples.

The Hereditary Breast and Ovarian Cancer Research Group Netherlands (HEBON): collaborating centers: Netherlands Cancer Institute, Amsterdam: MA Rookus, FBL Hogervorst, FE van Leeuwen, S Verhoef, MK Schmidt, JL de Lange and R Wijnands; Erasmus Medical Center, Rotterdam, the Netherlands: JM Collée, AMW van den Ouweland, MJ Hooning, C Seynaeve, CHM van Deurzen and IM Obdeijn; Leiden University Medical Center, Leiden, the Netherlands: CJ van Asperen, JT Wijnen, RAEM Tollenaar, P Devilee and TCTEF van Cronenburg; Radboud University Nijmegen Medical Center, Nijmegen, the Netherlands: CM Kets and AR Mensenkamp; University Medical Center Utrecht, Utrecht, the Netherlands: MGEM Ausems and RB van der Luijt; Amsterdam Medical Center, Amsterdam: CM Aalfs and TAM van Os; VU University Medical Center, Amsterdam: JJP Gille, Q Waisfisz and HEJ Meijers-Heijboer; University Hospital Maastricht, Maastricht, the Netherlands: EB Gómez-Garcia and MJ Blok; University Medical Center Groningen, Groningen, the Netherlands: JC Oosterwijk, AH van der Hout, MJ Mourits and GH de Bock; The Netherlands Foundation for the Detection of Hereditary Tumors, Leiden, the Netherlands: HF Vasen; The Netherlands Cancer Registry: S Siesling; The Dutch Pathology Registry (PALGA): LIH Overbeek; Hong Kong Sanatorium \& Hospital for their continual support; Janos Papp, Tibor Vaszko, Aniko Bozsik, Timea Pocza, Judit Franko, Maria Balogh, Gabriella Domokos, Judit Ferenczi (Department of Molecular Genetics, National Institute of Oncology, Budapest, Hungary) and the clinicians and patients for their contributions to this study; the Oncogenetics Group and the High Risk and Cancer Prevention Unit of the University Hospital Vall d'Hebron, Barcelona, Spain, led by Dr J Balmaña; the ICO Hereditary Cancer Program team led by Dr Gabriel Capella; Dr Martine Dumont and Martine Tranchant for sample management and skillful technical assistance; JS and PS were part of the quality control and genotyping coordinating group of iCOGS (BCAC and CIMBA); Drs Ana Peixoto, Catarina Santos, Patrícia Rocha and Pedro Pinto for their skillful contributions to the study; Heather Thorne, Eveline Niedermayr, all the kConFab research nurses and staff, the heads and staff of the Family Cancer Clinics, and the clinical follow-up study (which has received funding from the National Health and Medical Research Council (NHMRC), the National Breast Cancer Foundation, Cancer Australia and the National Institutes of Health (Bethesda, MD, USA)) for their contributions to this resource; and the many families who contribute to Kathleen Cuningham Foundation Consortium for Research into Familial Breast Cancer (kConFab); Lenka Foretova and Eva Machackova (Department of Cancer Epidemiology and Genetics, Masaryk Memorial Cancer Institute, and the Medical Faculty, Masaryk University, Brno, 
Czech Republic); Michal Zikan, Petr Pohlreich and Zdenek Kleibl (Oncogynecologic Center and Department of Biochemistry and Experimental Oncology, First Faculty of Medicine, Charles University, Prague, Czech Republic); Anne Lincoln and Lauren Jacobs; the National Israeli Cancer Control Center (NICCC) National Familial Cancer Consultation Service team led by Sara Dishon; the laboratory team led by Dr Flavio Lejbkowicz; and the research field operations team led by Dr Mila Pinchev; members and participants in the Ontario Cancer Genetics Network for their contributions to the study. Leigha Senter, Kevin Sweet, Caroline Craven and Michelle O'Conor were instrumental in accrual of study participants, ascertainment of medical records and database management; The Ohio State University Human Genetics Sample Bank; the Meirav Breast Center team at the Sheba Medical Center; Åke Borg, Håkan Olsson, Helena Jernström, Karin Henriksson, Katja Harbst, Maria Soller and Ulf Kristoffersson; Sahlgrenska University Hospital, Gothenburg, Sweden: Anna Öfverholm, Margareta Nordling, Per Karlsson and Zakaria Einbeigi; Stockholm and Karolinska University Hospital, Stockholm: Anna von Wachenfeldt, Annelie Liljegren, Annika Lindblom, Brita Arver, Gisela Barbany Bustinza and Johanna Rantala; Umeå University Hospital, Umeå, Sweden: Beatrice Melin, Christina Edwinsdotter Ardnor and Monica Emanuelsson; Uppsala University, Uppsala, Sweden: Hans Ehrencrona, Maritta Hellström Pigg and Richard Rosenquist; Linköping University Hospital, Linköping, Sweden: Marie Stenmark-Askmalm and Sigrun Liedgren; Cecilia Zvocec, Qun Niu, and physicians, genetic counselors, research nurses and staff of the Comprehensive Cancer Risk and Prevention Clinic of University of Chicago Medicine, Chicago, IL, USA, for their contributions to this resource; Joyce Seldon, MSGC, and Lorna Kwan, $\mathrm{MPH}$; Dr Robert Nussbaum and the following genetic counselors: Beth Crawford, Kate Loranger, Julie Mak, Nicola Stewart, Robin Lee, Amie Blanco and Peggy Conrad; Salina Chan; Paul DP Pharoah, Simon Gayther, Susan Ramus, Carole Pye, Patricia Harrington and Eva Wozniak for their contributions to the UK Familial Ovarian Cancer Registry (UKFOCR); Geoffrey Lindeman, Marion Harris, Martin Delatycki of the Victorian Familial Cancer Trials Group; and Sarah Sawyer, Rebecca Driessen and Ella Thompson.

\section{Funding}

Higher-level funding: The COGS project is funded through a European Commission Seventh Framework Program grant (agreement number 223175: HEALTH-F2-2009-223175). The CIMBA data management and data analysis were supported by Cancer Research UK grants C12292/A11174 and C1287/A10118. SH is supported by a National Health and Medical Research Council (NHMRC) program grant (to GCT).

Individual researcher support: ACA is a Cancer Research UK Senior Cancer Research Fellow (C12292/A11174). DFE is a Principal Research Fellow of Cancer Research UK. GC, MCS and IC are supported by the National Health and Medical Research Council (NHMRC). BK holds an American Cancer Society Early Detection Professorship (SIOP-06-258-01-COUN). MHG and PLM were supported by funding from the Intramural Research Program of the National Cancer Institute, National Institutes of Health. OlO is an American Cancer Society Clinical Research Professor. JS is Chairholder of the Canada Research Chair in Oncogenetics.

Funding of constituent studies: The Breast Cancer Family Registry (BCFR) was supported by grant UM1 CA164920 from the National Cancer Institute, National Institutes of Health. The content of this article does not necessarily reflect the views or policies of the National Cancer Institute or any of the collaborating centers in the BCFR, nor does mention of trade names, commercial products or organizations imply endorsement by the US Government or the BCFR. The Baltic Familial Breast Ovarian Cancer Consortium (BFBOCC) is partly supported by Lithuania (BFBOCC-LT): Research Council of Lithuania grant LIG-07/2012; BFBOCC-LV (Latvia) is partly supported by LSC grant 10.0010 .08 and in part by a grant from the European Social Fund number 2009/0220/1DP/1.1.1.2.0/09/APIANIAA/016 and the Liepāja City Council, Liepāja, Latvia; Beth Israel Deaconess Medical Center Cancer Center is supported by the Breast Cancer Research Foundation; $B R C A$-gene mutations and breast cancer in South African women (BMBSA) was supported by grants from the Cancer Association of South Africa (CANSA) to Elizabeth J van Rensburg; SLN (Beckman Research Institute, City of Hope, Duarte, CA, USA) was partially supported by the Morris and Horowitz Families Professorship in Cancer Etiology and Outcomes Research; the Copenhagen Breast Cancer Study (CBCS) was supported by the NEYE Foundation; the Spanish National Cancer Research Center (Centro Nacional de Investigaciones Oncológicas (CNIO)) was partially supported by the Spanish Association against Cancer (Asociación Española Contra el Cáncer AECC08), Thematic Network
Cooperative Research in Cancer (Red Temática Investigación Cooperativa en Cáncer (RTICC), Centro de Investigación Cáncer, Salamanca, Spain) RTICC 06/0020/1060, Spanish Ministry of Science and Innovation grants FIS PI08 1120 (Fondo de Investigación Sanitaria (FIS)) and SAF2010-20493, and the Fundación Mutua Madrileña (FMMA); the City of Hope Clinical Cancer Genetics Community Network and the Hereditary Cancer Research Registry (COH-CCGCRN), supported in part by award number RC4CA153828 (Principal Investigator: JNW) from the National Cancer Institute and the Office of the Director, National Institutes of Health. The content is solely the responsibility of the authors and does not necessarily represent the official views of the National Institutes of Health. CONsorzio Studi ITaliani sui Tumori Ereditari Alla Mammella, Italy (CONSIT Team): Funds from Italian citizens who allocated the " $5 \times 1,000$ " share of their tax payment in support of the Fondazione IRCCS Istituto Nazionale Tumori, according to Italian laws (Istituto Nazionale dei Tumori (INT) institutional strategic project " $5 \times 1,000$ ") (to SM); the Italian Association for Cancer Research (AIRC) (to LO); National Centre for Scientific Research "Demokritos" has been cofinanced by the European Union (European Social Fund (ESF)) and Greek national funds through the "Education and Lifelong Learning" operational program of the National Strategic Reference Framework (NSRF) - Research Funding Program of the General Secretariat for Research and Technology: ARISTEIA; "Heracleitus II: Investing in knowledge society through the European Social Fund"; the DKFZ study was supported by the Deutsches Krebsforschungszentrum (DKFZ); Epidemiological Study of BRCA1 and BRCA2 Mutation Carriers (EMBRACE) is supported by Cancer Research UK grants C1287/A10118 and C1287/A11990; DGE and FL are supported by a National Institute for Health Research (NIHR) grant to the Biomedical Research Centre, Manchester, UK; the investigators at The Institute of Cancer Research and The Royal Marsden NHS Foundation Trust are supported by an NIHR grant to the Biomedical Research Centre at The Institute of Cancer Research and The Royal Marsden NHS Foundation Trust, London; RE and EB are supported by Cancer Research UK grant C5047/A8385; Kansas University Medical Center: The authors acknowledge support from The University of Kansas Cancer Center (P30 CA168524) and the Kansas Bioscience Authority Eminent Scholar Program; AKG was funded by grants 5 U01 CA113916 and R01 CA140323 and by the Chancellors Distinguished Chair in Biomedical Sciences Professorship; The German Consortium of Hereditary Breast and Ovarian Cancer (GC-HBOC) is supported by German Cancer Aid (grant 109076 (to RKS) and by the Center for Molecular Medicine Cologne (CMMC); GC-HBOC is deeply grateful to Dr Sabine Preisler-Adam for providing information and samples; the GEMO Study was supported by the Ligue National Contre le Cancer; the Association "Le cancer du sein, parlons-en!" Award; and the Canadian Institutes of Health Research for the CIHR Team in Familial Risks of Breast Cancer program; G-FAST: KDL is supported by GOA grant BOF10/GOA/019 (Ghent University) and spearhead financing of Ghent University Hospital; the Gynecologic Oncology Group (GOG) was supported by National Cancer Institute grants to the GOG Administrative Office and Tissue Bank (grant CA 27469), the GOG Statistical and Data Center (grant CA 37517) and GOG's Cancer Prevention and Control Committee (grant CA 101165); HCSC was supported by grants RD12/00369/0006 and 12/00539 from Instituto de Salud Carlos III (ISCIII), Madrid, Spain, partially supported by European Regional Development Fund (Fonds européen de développement régional (FEDER)) funds; the Helsinki Breast Cancer Study (HEBCS) was financially supported by the Helsinki University Central Hospital Research Fund, Academy of Finland (266528), the Finnish Cancer Society and the Sigrid Juselius Foundation; HEBON is supported by the Dutch Cancer Society grants NKI1998-1854, NKI2004-3088 and NKI2007-3756, the Netherlands Organization of Scientific Research grant NWO 91109024, the Pink Ribbon grant 110005 and Biobanking and Molecular Resource Infrastructure (BBMRI) grant NWO 184.021.007/CP46; HEBON thanks the registration teams of the Comprehensive Cancer Centre Netherlands and Comprehensive Centre South (together the Netherlands Cancer Registry) and PALGA (Dutch Pathology Registry) for part of the data collection; the High Risk Breast Cancer Program (HRBCP) is supported by the Hong Kong Hereditary Breast Cancer Family Registry and the Dr Ellen Li Charitable Foundation, Hong Kong; the Hungarian Breast and Ovarian Cancer Study (HUNBOCS) was supported by Hungarian Research and Technological Innovation Fund (KTIA)/Hungarian Scientific Research Fund (Országos Tudományos Kutatási Alapprogramok (OTKA)) research grants KTIA-OTKA CK-80745 and KTIA-OTKA K-112228; Institut Català d'Oncologia (ICO): contract grant sponsor: Asociación Española Contra el Cáncer; Spanish Health Research Foundation; Ramón Areces Foundation; Instituto de Salud 
Carlos III (ISCIII); Catalan Health Institute; and Autonomous Government of Catalonia; contract grant numbers ISCIIIRETIC RD06/0020/1051, PI09/02483, PI10/01422, PI10/00748, PI13/00285, PI13/00189 2009SGR290 and PI13/00189 2009SGR283; the International Hereditary Cancer Center (Department of Genetics and Pathology, Pomeranian Medical University, Szczecin, Poland) was supported by grant PBZ_KBN_122/P05/2004; Landspitali - The National University Hospital of Iceland was supported by the Icelandic Association "Walking for Breast Cancer Research" and by the Landspitali University Hospital Research Fund; the Interdisciplinary Health Research Internal Team Breast Cancer Susceptibility Study (INHERIT) was supported by the Canadian Institutes of Health Research (CIHR) for the "CIHR Team in Familial Risks of Breast Cancer" program, Canadian Breast Cancer Research Alliance grant 019511 and Ministry of Economic Development, Innovation and Export Trade grant PSR-SIIRI-701; the Istituto Oncologico Veneto Hereditary Breast and Ovarian Cancer Study (IOVHBOCS) is supported by the Ministero della Salute and a " $5 \times 1,000$ " Istituto Oncologico Veneto grant; the Portuguese Oncology Institute-Porto Breast Cancer Study (IPOBCS) was supported in part by Liga Portuguesa Contra o Cancro; kConFab is supported by a grant from the National Breast Cancer Foundation and previously by the National Health and Medical Research Council (NHMRC), the Queensland Cancer Fund, the Cancer Councils of New South Wales, Victoria, Tasmania and South Australia, and the Cancer Foundation of Western Australia; the Mayo Clinic is supported by National Institutes of Health (NIH) grants CA 116167, CA 128978 and CA 176785 through the National Cancer Institute (NCI), an NCl Specialized Program of Research Excellence (SPORE) in Breast Cancer (CA116201), a US Department of Defense Ovarian Cancer Idea award (W81XWH-10-1-0341), a grant from the Breast Cancer Research Foundation, a generous gift from the David F and Margaret T Grohne Family Foundation and the Ting Tsung and Wei Fong Chao Foundation; McGill University Jewish General Hospital Weekend to End Breast Cancer, Quebec Ministry of Economic Development, Innovation and Export Trade; Modifier Study of Quantitative Effects on Disease (ModSQuaD) was supported by the Ministry of Health of the Czech Republic to Masaryk Memorial Cancer Institute ( $\mathrm{MH}$ CZ - DRO) (MMCl 00209805) and by the European Regional Development Fund and the State Budget of the Czech Republic (RECAMO, CZ.1.05/ 2.1.00/03.0101) (to LF), and by Charles University in Prague project UNCE204024 (MZ); Memorial Sloan Kettering Cancer Center (MSKCC) is supported by grants from the Breast Cancer Research Foundation and Robert and Kate Niehaus Clinical Cancer Genetics Initiative; National Cancer Institute, National Institutes of Health: The research of MHG and PLM was supported by the Intramural Research Program of the National Cancer Institute and by support services contracts N02-CP-11019-50 and N02-CP-65504 with Westat, Inc, Rockville, MD, USA; the National Israeli Cancer Control Center (NICCC) is supported by Clalit Health Services in Israel; some of its activities are supported by the Israel Cancer Association and the Breast Cancer Research Foundation (BCRF), New York, NY, USA; NN Petrov Institute of Oncology has been supported by the Russian Federation for Basic Research (grants 11-04-00227, 12-04-00928 and 12-04-01490) and the Federal Agency for Science and Innovations, Russia (contract 02.740.11.0780); The Ohio State University Clinical Cancer Genetics (OSUCCG) is supported by The Ohio State University Comprehensive Cancer Center; the Pisa Breast Cancer Study (PBCS) was supported by Istituto Toscano Tumori (ITT) grants 2011-2013; Sheba Medical Center was partially funded through a grant from the Israel Cancer Association and funding for the Israeli Inherited Breast Cancer Consortium; the Swedish Breast Cancer Study collaborators are supported by the Swedish Cancer Society; The University of Chicago is supported by NCI Specialized Program of Research Excellence (SPORE) in Breast Cancer (CA125183), R01 CA142996 and 1U01CA161032 and by the Ralph and Marion Falk Medical Research Trust, the Entertainment Industry Fund National Women's Cancer Research Alliance and the Breast Cancer Research Foundation; University of California, Los Angeles Jonsson Comprehensive Cancer Center Foundation: Breast Cancer Research Foundation; University of California, San Francisco Cancer Risk Program and Helen Diller Family Comprehensive Cancer Center; UK Familial Ovarian Cancer Registry (UKFOCR) was supported by a project grant from Cancer Research UK (to Paul DP Pharoah); the University of Pennsylvania: National Institutes of Health (NIH) grants R01 CA102776 and R01 CA083855; the Breast Cancer Research Foundation; the Susan G Komen for the Cure, Basser Center for BRCA; Victorian Familial Cancer Trials Group (VFCTG): Victorian Cancer Agency, Cancer Australia, National Breast Cancer Foundation; The Women's Cancer Program (WCP) at the Samuel Oschin Comprehensive Cancer Institute, Cedars-Sinai Medical Center, is funded by the American Cancer Society Early Detection Professorship (SIOP-06-258-01-COUN).

GEMO Study: National Cancer Genetics Network UNICANCER Genetic Group, France.

The Hereditary Breast and Ovarian Cancer Research Group Netherlands (HEBON) coordinating center: Netherlands Cancer Institute, Amsterdam.

\section{Author details}

${ }^{1}$ INSERM U1052, CNRS UMR5286, Université Lyon 1, Centre de Recherche en Cancérologie de Lyon, Lyon, France. ${ }^{2}$ Université de Lyon, 69000 Lyon, France. ${ }^{3}$ Université Lyon 1, 69100 Villeurbanne, France. ${ }^{4}$ UMR CNRS 5558, Laboratoire de Biométrie et Biologie Évolutive (LBBE), "Biométrie et Biologie Évolutive", Université Claude Bernard Lyon 1, Bâtiment Grégor Mendel, 43 boulevard du 11 novembre 1918, 69622 Villeurbanne, cedex, France. ${ }^{5}$ Université Grenoble Alpes, UMR 5217, Laboratoire d'Informatique de Grenoble (LIG), équipe-projet Multi-programmation et Ordonnancement sur ressources pour les Applications Interactives de Simulation (MOAIS), 38041 Grenoble, France. ${ }^{6}$ INRIA Rhône-Alpes, équipe-projet MOAIS, 38334 Saint Ismier, Cedex, France. ${ }^{7}$ Centre for Cancer Genetic Epidemiology, Department of Public Health and Primary Care, University of Cambridge, Cambridge, UK. ${ }^{8}$ Department of Genetics and Computational Biology, QIMR Berghofer, Brisbane, Australia. ${ }^{9}$ Centre de recherche du Centre hospitalier universitaire de Québec, Laval University, Charlesbourg, PQ, Canada. ${ }^{10}$ Department of Epidemiology, Mailman School of Public Health, Columbia University, New York, NY, USA. ${ }^{11}$ Department of Pediatrics, Columbia University College of Physicians and Surgeons, New York, NY, USA. ${ }^{12}$ Department of Medicine, Columbia University College of Physicians and Surgeons, New York, NY, USA. ${ }^{13}$ Department of Dermatology, University of Utah School of Medicine, Salt Lake City, UT, USA. ${ }^{14}$ Department of Internal Medicine, Huntsman Cancer Institute, University of Utah School of Medicine, Salt Lake City, UT, USA. ${ }^{15}$ Department of Epidemiology, Cancer Prevention Institute of California, 2201 Walnut Avenue, Suite 300, Fremont, CA 94538, USA. ${ }^{16}$ Hematology, Oncology and Transfusion Medicine Center, Vilnius University Hospital Santariskiu Clinics, Vilnius, Lithuania. ${ }^{17}$ Department of Molecular and Regenerative Medicine, Centre for Innovative Medicine, State Research Institute, Vilnius, Lithuania. ${ }^{18}$ Latvian Biomedical Research and Study Centre, Rātsupītes iela 1, Rīga LV-1067, Latvia. ${ }^{19}$ Division of Hematology Oncology, Beth Israel Deaconess Medical Center, 330 Brookline Avenue, Shapiro 9, Boston, MA 02215-5400, USA. ${ }^{20}$ Department of Genetics, University of Pretoria, Private Bag X20, Hatfield, 0028 Pretoria, South Africa. ${ }^{21}$ Department of Population Sciences, Beckman Research Institute, City of Hope, 1500 East Duarte Road, Duarte, CA 91010, USA. ${ }^{22}$ Department of Clinical Genetics, Rigshospitalet, Copenhagen University Hospital, Copenhagen, Denmark. ${ }^{23}$ Department of Oncology, Rigshospitalet, Copenhagen University Hospital, Copenhagen, Denmark. ${ }^{24}$ Center for Genomic Medicine, Rigshospitalet, Copenhagen University Hospital, Copenhagen, Denmark. ${ }^{25}$ Human Genetics Group, Spanish National Cancer Research Center (CNIO), Madrid, Spain. ${ }^{26}$ Center for Biomedical Network Research on Rare Diseases (CIBERER), Madrid, Spain. ${ }^{27}$ Medical Oncology Service, Hospital Clínico Universitario Lozano Blesa, Avenida San Juan Bosco, 15, 50009 Zaragoza, Spain. ${ }^{28}$ Holy Cross Hospital, Michael and Dianne Bienes Comprehensive Cancer Center, Fort Lauderdale, FL, USA. ${ }^{29}$ Division of Clinical Cancer Genetics, City of Hope (for the Clinical Cancer Genetics Community Research Network), City of Hope, 1500 East Duarte Road, Duarte, CA 91010, USA. ${ }^{30} J o h n$ Muir Medical Center, Walnut Creek, CA, USA; c/o Clinical Cancer Genetics Community Research Network, City of Hope, 1500 East Duarte Road, Duarte, CA 91010, USA. ${ }^{31}$ Istituto FIRC di Oncologia Molecolare (IFOM), Via Adamello 16, 20139 Milan, Italy. ${ }^{32}$ Unit of Molecular Bases of Genetic Risk and Genetic Testing, Department of Preventive and Predictive Medicine, Istituto di Ricovero e Cura a Carattere Scientifico (IRCCS), Istituto Nazionale dei Tumori (INT), Via Venezian 1, 20133 Milan, Italy. ${ }^{33}$ Cogentech Cancer Genetic Test Laboratory, Via Adamello 16, 20139 Milan, Italy. ${ }^{34}$ Cancer Bioimmunotherapy Unit, Centro di Riferimento Oncologico (CRO), Via Franco Gallini 2, 33081 Aviano, Italy. ${ }^{35}$ Division of Cancer Prevention and Genetics, Istituto Europeo di Oncologia, Via Ripamonti 435, 20141 Milan, Italy. ${ }^{36}$ Unit of Medical Genetics, Department of Preventive and Predictive Medicine, Istituto di Ricovero e Cura a Carattere Scientifico (IRCCS), Istituto Nazionale dei Tumori (INT), Via Venezian 1, 20133 Milan, Italy. ${ }^{37}$ Unit of Hereditary Cancer, Department of Epidemiology, 
Prevention and Special Functions, Istituto di Ricovero e Cura a Carattere Scientifico (IRCCS), Azienda Ospedaliera Universitaria "San Martino" di Genova, IST Istituto Nazionale per la Ricerca sul Cancro, Largo Rosanna Benzi, 10, 16132 Genoa, Italy. ${ }^{38}$ FiorGen Foundation for Pharmacogenomics, Via Luigi Sacconi 6, 50019 Sesto Fiorentino, Italy. ${ }^{39}$ Unit of Medical Genetics, Department of Biomedical, Experimental and Clinical Sciences, University of Florence, Florence, Italy. ${ }^{40}$ Department of Molecular Medicine, Sapienza University, Rome, Italy. ${ }^{41}$ Department of Medical Oncology, Papageorgiou Hospital, Aristotle University of Thessaloniki School of Medicine, Thessaloniki, Greece. ${ }^{42}$ Molecular Diagnostics Laboratory, INRASTES, National Centre for Scientific Research "Demokritos", Aghia Paraskevi Attikis, Athens, Greece. ${ }^{43}$ Dana-Farber Cancer Institute, 450 Brookline Avenue, Boston, MA 02215, USA. ${ }^{44}$ Molecular Genetics of Breast Cancer, Deutsches Krebsforschungszentrum (DKFZ), Heidelberg, Germany. ${ }^{45}$ Clinical Genetics Department, St Michael's Hospital, Southwell Street, Bristol BS2 8EG, UK. ${ }^{46}$ North West Thames Regional Genetics Service, Kennedy-Galton Centre, Harrow, UK. ${ }^{47}$ Department of Clinical Genetics, Royal Devon \& Exeter Hospital, Barrack Road, Exeter EX2 5DW, UK. ${ }^{48}$ Merseyside and Cheshire Clinical Genetics Service, Liverpool Women's NHS Foundation Trust, Crown Street, Liverpool, Merseyside L8 7SS, UK. ${ }^{49}$ Genetic Medicine, Manchester Academic Health Sciences Centre, Central Manchester University Hospitals NHS Foundation Trust, Manchester, UK. ${ }^{50}$ Centre for Cancer Genetic Epidemiology, Department of Public Health and Primary Care, Strangeways Research Laboratory, University of Cambridge, Worts Causeway, Cambridge CB1 8RN, UK. ${ }^{51}$ Faculty of Medicine, University of Southampton, Southampton University Hospitals NHS Trust, Mailpoint 801, South Academic Block, PAH/G/MP105, Tremona Road, Southampton SO16 6YD, UK. ${ }^{52}$ Institute of Human Genetics, Northern Genetic Service, International Centre for Life, Newcastle upon Tyne Hospitals NHS Trust, Central Parkway, Newcastle upon Tyne NE1 4EP, UK. ${ }^{53}$ Sheffield Clinical Genetics Service, Sheffield Children's Hospital, Sheffield, UK ${ }^{54}$ Yorkshire Regional Genetics Service, Leeds Teaching Hospitals NHS Trust, Old Medical School, Leeds General Infirmary, Leeds LS1 3EX, UK. ${ }^{55}$ Leicestershire Clinical Genetics Service, Department of Clinical Genetics, Leicester Royal Infirmary, University Hospitals of Leicester NHS Trust, Leicester LE1 5WW, UK. ${ }^{56}$ Oxford Regional Genetics Service, Churchill Hospital, Old Road, Headington, Oxford OX3 7LE, UK. ${ }^{57}$ Clinical Genetics Service, Guy's and St Thomas' NHS Foundation Trust, 7th floor, Borough Wing, Guy's Hospital, Great Maze Pond, London SE1 9RT, UK. ${ }^{58}$ North East Thames Regional Genetics Service, Great Ormond Street Hospital for Children NHS Trust, Barclay House, 37, Queen Square, London WC1N 3BH, UK. ${ }^{59}$ Academic Unit of Clinical and Molecular Oncology, Trinity College Dublin, College Green, Dublin 2, Ireland. ${ }^{60}$ Medical Oncology Service, St James's Hospital, James's Street, Dublin 8, Ireland. ${ }^{61}$ Department of Clinical Genetics, East Anglian Regional Genetics Service, Addenbrooke's Hospital, Level 6, Addenbrooke's Treatment Centre, Cambridge University Hospitals NHS Foundation Trust, Hills Road, Cambridge CB2 0QQ, UK ${ }^{62}$ All Wales Medical Genetics Services, University Hospital of Wales, Heath Park, Cardiff CF14 4XW, UK. ${ }^{63}$ South East Scotland Regional Genetic Service, Western General Hospital, David Brock Building, Crewe Road South, Edinburgh EH4 2XU, UK. ${ }^{64}$ Centre for Cancer Research \& Cell Biology, School of Medicine, Dentistry and Biomedical Sciences, Queen's University Belfast, 97 Lisburn Road, Belfast BT9 $7 A E$, UK. ${ }^{65}$ Department of Medical Genetics, Belfast Health and Social Care Trust, Belfast City Hospital, Lisburn Road, Belfast BT9 7AB, UK. ${ }^{66}$ Oncogenetics Team, The Institute of Cancer Research and Royal Marsden NHS Foundation Trust, 123 Old Brompton Road, London SW7 3RP, UK. ${ }^{67}$ Ferguson-Smith Centre for Clinical Genetics, Yorkhill Hospitals, Block 4, Glasgow G3 8SJ, UK ${ }^{68}$ South West Thames Regional Genetics Service, Department of Medical Genetics, St George's University of London, Cranmer Terrace, London SW17 ORE, UK. ${ }^{69}$ West Midlands Regional Genetics Service, Birmingham Women's Hospital Healthcare NHS Trust, Mindelsohn Way, Edgbaston, Birmingham B15 2TG, UK. ${ }^{70}$ Department of Pathology \& Laboratory Medicine, University of Kansas Medical Center, 3901 Rainbow Boulevard, Kansas City, KS 66160, USA. ${ }^{71}$ Lombardi Comprehensive Cancer Center, MedStar Georgetown University Hospital, 3800 Reservoir Road NW, Washington, DC 20057, USA. ${ }^{72}$ Center for Medical Genetics, Ghent University Hospital, De Pintelaan 185, 9000 Ghent, Belgium. ${ }^{73}$ Division of Tumor Genetics, Department of Gynaecology and Obstetrics, University Hospital Klinikum Rechts der Isar, Technische Universität München, Ismaninger Strasse 22, 81675 Munich, Germany. ${ }^{74}$ Center of Familial Breast and Ovarian Cancer, Department of Medical Genetics, Institut für Humangenetik, Biozentrum, Universität Würzburg, Am Hubland, 97074 Würzburg, Germany. ${ }^{75}$ Center for Hereditary Breast and Ovarian Cancer, Medical Faculty, Center for Integrated Oncology (CIO) Cancer Center Cologne, University Hospital Cologne, Cologne, Germany. ${ }^{76}$ Center for Molecular Medicine Cologne (CMMC), University of Cologne, Robert-Koch-Strasse 21, 50931
Cologne, Germany. ${ }^{77}$ Department of Human Genetics, Institute of Human Genetics, University Hospital Heidelberg, Heidelberg, Germany. ${ }^{78}$ Institute for Medical Informatics, Statistics and Epidemiology, Medical Faculty, University of Leipzig, Leipzig, Germany. ${ }^{79}$ Department of Gynaecology and Obstetrics, University Hospital Düsseldorf, Heinrich-Heine University Düsseldorf, Moorenstrasse 5, 40225 Düsseldorf, Germany. ${ }^{80}$ Institute of Cell and Molecular Pathology, Centre for Pathology and Forensic and Genetic Medicine, Hannover Medical School, Carl-Neuberg-Strasse 1, 30625 Hannover, Germany. ${ }^{81}$ Institute of Human Genetics, University Medical Center Schleswig-Holstein, Campus Kiel, Arnold-Heller-Strasse 3, D-24105 Kiel, Germany. ${ }^{82}$ Department of Gynecology and Obstetrics, University Hospital Carl Gustav Carus of Dresden, Technical University Dresden, Dresden, Germany. ${ }^{83}$ Department of Gynecology and Obstetrics, University Medical Center Schleswig-Holstein, Campus Kiel, Arnold-Heller-Strasse 3, D-24105 Kiel, Germany. ${ }^{84}$ Institute of Medical Genetics and Human Genetics, Campus Virchow-Klinikum, Charité Berlin - Universtitätsmedizin Berlin, Augustenburger Platz 1, 13353 Berlin, Germany. ${ }^{85}$ German Consortium of Hereditary Breast and Ovarian Cancer (GC-HBOC), Cologne, Germany. ${ }^{86}$ Institute of Human Genetics, University Hospital Münster, Vesaliusweg 12-14, 48149 Münster, Germany. ${ }^{87}$ Department of Gynecology and Obstetrics, University Hospital UIm, Ulm, Germany. ${ }^{88}$ Department of Tumour Biology, Institut Curie, 26 rue d'Ulm 75248, Paris cedex 05, France. ${ }^{89}$ Unité de Prévention et d'Épidémiologie Génétique, Centre Léon Bérard, 28 rue Laenned, 69008 Lyon, France. ${ }^{90}$ Génétique Clinique, Centre Hospitalier Universitaire de Grenoble, CS 10217, 38043, Grenoble cedex 9, France. ${ }^{91}$ Institut Albert Bonniot - Inserm U823, Université Joseph Fourier, Rond-point de la Chantourne, 38706 La Tronche, France. ${ }^{92}$ Laboratoire d'Oncogénétique, Hôpital René Huguenin, Institut Curie, 35 rue Dailly, 92210 Saint-Cloud, France. ${ }^{93}$ Département d'Oncogénétique, Centre Jean Perrin, Université de Clermont-Ferrand, 58 rue Montalembert, BP 392, 63011 ClermontFerrand, France. ${ }^{94}$ Gynaecological Oncology, Sydney Cancer Centre, Royal Prince Alfred Hospital and University of Sydney, Missenden Road, Camperdown, NSW 2050, Australia. ${ }^{95}$ Gynecologic Oncology Group, Department of OB-GYN, University of North Carolina at Chapel Hill, 103B Physicians' Office Building, CB\# 7572, Chapel Hill, NC 27599-7572, USA. ${ }^{96}$ Gynecologic Oncology Group Statistical and Data Center, Roswell Park Cancer Institute, Elm and Carlton Streets, Buffalo, NY 14263-0001, USA. ${ }^{97}$ Women \& Infants Hospital, 1 Blackstone Place, Providence, RI 02905, USA. ${ }^{98}$ Molecular Oncology Laboratory, Health Research Institute of the San Carlos Clinical Hospital (IdISSC), 28040 Madrid, Spain. ${ }^{99}$ Department of Obstetrics and Gynecology, University of Helsinki and Helsinki University Central Hospital, Biomedicum Helsinki, PO Box 700, 00029 Helsinki, Finland.

${ }^{100}$ Department of Clinical Genetics, Helsinki University Central Hospital, Biomedicum Helsinki 1, Haartmaninkatu 8, 00290 Helsinki, Finland. ${ }^{101}$ Department of Medical Oncology, Family Cancer Clinic, Erasmus University Medical Center, PO Box 2040, 3000 CA, Rotterdam, the Netherlands. ${ }^{102}$ Department of Clinical Genetics, Family Cancer Clinic, Erasmus University Medical Center, Rotterdam, the Netherlands. ${ }^{103}$ Department of Human Genetics, Radboud University Nijmegen Medical Centre, Nijmegen, the Netherlands. ${ }^{104}$ Department of Clinical Genetics, Academic Medical Center, Amsterdam, the Netherlands. ${ }^{105}$ Department of Epidemiology, Netherlands Cancer Institute, Amsterdam, the Netherlands. ${ }^{106}$ Family Cancer Clinic, Netherlands Cancer Institute, Amsterdam, the Netherlands. ${ }^{107}$ Department of Clinical Genetics, VU University Medical Center Amsterdam, De Boelelaan 1118, 1081 HV Amsterdam, the Netherlands. ${ }^{108}$ Department of Genetics, University Medical Center, Groningen University, Groningen, the Netherlands. ${ }^{109}$ Department of Clinical Genetics, Maastricht University Medical Center, Maastricht, the Netherlands. ${ }^{110}$ Department of Human Genetics, Center for Human and Clinical Genetics, Leiden University Medical Center, S4-P PO Box 9600, 2300 RC, Leiden, the Netherlands. ${ }^{11}$ Department of Pathology, Leiden University Medical Center, PO Box 9600, 2300 RC L1Q Leiden, the Netherlands. ${ }^{112}$ Department of Medical Genetics, University Medical Center Utrecht, Utrecht, the Netherlands. ${ }^{113}$ Department of Molecular Genetics, National Institute of Oncology, Ráth György u 7-9, PO Box 1525 Budapest PF 211122 Budapest, Hungary. ${ }^{114}$ Oncogenetics Group, Vall d'Hebron Institute of Oncology (VHIO), University Hospital Vall d'Hebron, Vall d'Hebron Research Institute (VHIR) and Universitat Autònoma de Barcelona, Passeig de la Vall d'Hebron 119, 08035 Barcelona, Spain. ${ }^{115}$ Genetic Counseling Unit, Hereditary Cancer Program, Institut d'Investigació Biomèdica de Bellvitge (IDIBELL)-Catalan Institute of Oncology, Hospital Duran i Reynals, 3a planta - Gran Via de I'Hospitalet, 199, 08908 Hospitalet de Llobregat, Barcelona, Spain. ${ }^{116}$ Molecular Diagnostic Unit, Hereditary Cancer Program, Institut d'Investigació Biomèdica de Bellvitge (IDIBELL)-Catalan Institute of Oncology, Hospital Duran i Reynals, 3a planta - Gran Via de l'Hospitalet, 199, 08908 Hospitalet de Llobregat, Barcelona, Spain. ${ }^{117}$ Department of Genetics and Pathomorphology, Faculty of Medicine and Dentistry, Pomeranian Medical 
University, al Powstancow W/kp 72, 70-111 Szczecin, Poland. ${ }^{118}$ Landspítali National University Hospital of Iceland and Faculty of Medicine, School of Health Sciences, University of Iceland School of Medicine, Sæmundargötu 2, 101 Reykjavik, Iceland. ${ }^{119}$ Laboratoire de diagnostic génétique et Service d'Onco-hématologie, Les Hopitaux Universitaire de Strasbourg, Nouvel Hôpital Civil, 1 place de l'Hôpital, BP 426, 67091 Strasbourg, France. ${ }^{120}$ Department of Surgical Sciences, Oncology and Gastroenterology, Padua University, Clinical Surgery II, via Giustiniani 2, 35124 Padua, Italy. ${ }^{121}$ Immunology and Molecular Oncology Unit, Istituto Oncologico Veneto (IOV) - Istituto di Ricovero e Cura a Carattere Scientifico (IRCCS), via Gattamelata 64, 35128 Padua, Italy. ${ }^{122}$ Department of Genetics, Portuguese Oncology Institute (IPO-PORTO), Edifício dos Laboratórios, piso 6, 4200-072 Porto, Portugal. ${ }^{123}$ Instituto de Ciências Biomédicas Abel Salazar (ICBAS), Instituto de Ciências Biomédicas Abel Salazar da Universidade do Porto, Rua de Jorge Viterbo Ferreira 228, 4050-313 Porto, Portugal. ${ }^{124}$ Program in Cancer Genetics, Departments of Human Genetics and Oncology, McGill University, 546 Pine Avenue West, Montreal, QC J2W 156, Canada. ${ }^{125}$ Department of Health Sciences Research, Mayo Clinic, 200 First Street SW, Rochester, MN 55905, USA. ${ }^{126}$ Department of Health Sciences Research, Mayo Clinic, 13400 East Shea Boulevard, Scottsdale, AZ 85259, USA. ${ }^{127}$ National Human Genome Research Institute, National Institutes of Health, Building 31, Room 4B09, 31 Center Drive, MSC 2152, 9000 Rockville Pike, Bethesda, MD 20892-2152, USA. ${ }^{128}$ Clinical Genetics Service, Department of Medicine, Memorial Sloan Kettering Cancer Center, 1275 York Avenue, New York, NY 10065, USA. ${ }^{129}$ Clinical Genetics Research Laboratory, Memorial Sloan Kettering Cancer Center, 1275 York Avenue, New York, NY 10065, USA. ${ }^{130}$ Department of Obstetrics and Gynecology, Comprehensive Cancer Center Vienna, Medical University of Vienna, Universitätsklinik für Frauenheilkunde, AKH - Wien, Währinger Gürtel 18-20, 1090 Vienna, Austria. ${ }^{131}$ Clinical Genetics Branch, Division of Cancer Epidemiology and Genetics, National Cancer Institute, National Institutes of Health, Bethesda, MD, USA. ${ }^{132}$ National Israeli Cancer Control Center and Department of Community Medicine and Epidemiology, Clalit Health Services Carmel Medical Center, 34361 Haifa, Israel. ${ }^{133}$ Ruth and Bruce Rappaport Faculty of Medicine, Technion - Israel Institute of Technology, 2 Horev Street, 34362 Haifa, Israel. ${ }^{134} \mathrm{NN}$ Petrov Institute of Oncology, 68 Leningradskaya Street, Pesochny 197758 St Petersburg, Russia. ${ }^{135}$ Department of Laboratory Medicine and Pathobiology, University of Toronto, Medical Sciences Building, 6th Floor, 1 King's College Circle, Toronto, ON M5S 1A8, Canada. ${ }^{136}$ Keenan Research Centre, Li Ka Shing Knowledge Institute, St Michael's Hospital, 209 Victoria Street, Toronto, ON M5B 1T8, Canada. ${ }^{137}$ Ontario Cancer Genetics Network, Cancer Care Ontario, 620 University Avenue, Toronto, ON M5G 2L7, Canada. ${ }^{138}$ Lunenfeld-Tanenbaum Research Institute, Mount Sinai Hospital Joseph and Wolf Lebovic Health Complex, 600 University Avenue, Toronto, ON M5G 1X5, Canada. ${ }^{139}$ Department of Molecular Genetics, University of Toronto, Medical Science Building, Room 4386, 1 King's College Circle, Toronto, ON M5S 1A8, Canada. ${ }^{140}$ Department of Human Cancer Genetics, 1093 Biomedical Research Tower, 460 West 12th Avenue, Columbus, OH 43210, USA. ${ }^{141}$ Department of Internal Medicine, The Ohio State University Wexner Medical Center, North Doan Tower, 395 West 12th Avenue, Columbus, OH 43210, USA. ${ }^{142}$ Department of Molecular Virology, Immunology and Medical Genetics, The Ohio State University Wexner Medical Center, 1093 Biomedical Research Tower, 460 West 12th Avenue, Columbus, OH 43210, USA. ${ }^{143}$ The Ohio State University Comprehensive Cancer Center - Arthur G James Cancer Hospital and Richard J Solove Research Institute (OSUCCC - James), 460 West 10th Avenue, Columbus, OH 43210, USA. ${ }^{144}$ Section of Molecular Diagnostics, Department of Biochemistry, Aalborg University Hospital, Hobrovej 18, 9000 Aalborg, Denmark. ${ }^{145}$ Department of Clinical Genetics, Odense University Hospital, Soenderboulevard 29, 5000 Odense C, Denmark. ${ }^{146}$ Department of Clinical Genetics, Aarhus University Hospital, Brendstrupgårdsvej 21 C, 8200 Aarhus $\mathrm{N}$, Denmark. ${ }^{147}$ Laboratorio di Genetica Oncologica, Divisione di Anatomia Patologica e di Diagnostica Molecolare ed Ultrastrutturale, Azienda Ospedaliero Universitaria Pisana - Ospedale S Chiara, via Roma 67, 56126 Pisa, Italy. ${ }^{148}$ Sheba Laboratory of Molecular Genetics, The Danek Gertner Institute of Human Genetics, Sheba Medical Center, Tel Hashomer, Ramat Gan, 52621 Tel Aviv, Israel. ${ }^{149}$ Institute of Oncology, Rivka Ziv Medical Center, Maimonides, 13100 Safed, Israel. ${ }^{150}$ Department of Cancer Genetics, Karolinska University Hospital, Solna L8:02, SE-171 76 Stockholm, Sweden. ${ }^{151}$ Oncology, Department of Radiation Sciences, Umeå University, SE-901 87 Umeå, Sweden. ${ }^{152}$ Department of Oncology-Pathology, Karolinska University Hospital, K7, Ärftlighetsmottagningen, Radiumhemmet, 17176 Stockholm, Sweden. ${ }^{153}$ Division of Oncology and Pathology, Department of Clinical Sciences, Lund University Hospital, Barngatan 2B, SE-221 85 Lund, Sweden.

${ }^{154}$ Department of Immunology, Genetics and Pathology, Rudbeck
Laboratory, Uppsala University, 75185 Uppsala, Sweden. ${ }^{155}$ Center for Clinical Cancer Genetics and Global Health, The University of Chicago, 5841 South Maryland Avenue, Chicago, IL 60637, USA. ${ }^{156}$ Department of Medicine and Genetics, University of California, San Francisco, CA, USA.

${ }^{157}$ Department of Preventive Medicine, Keck School of Medicine, University of Southern California, 1441 Eastlake Avenue, Norris Comprehensive Cancer Center, NOR-4435, Los Angeles, CA 90089-9175, USA. ${ }^{158}$ Department of Medicine, Abramson Cancer Center, Perelman School of Medicine, University of Pennsylvania, 3535 Market Street, Suite 750, Philadelphia, PA 19104-3309, USA. ${ }^{159}$ Department of Epidemiology and Biostatistics, Abramson Cancer Center, Perelman School of Medicine, University of Pennsylvania, 3535 Market Street, Suite 750, Philadelphia, PA 19104-3309, USA. ${ }^{160}$ Division of Cancer Medicine, Department of Breast Medical Oncology, The University of Texas MD Anderson Cancer Center, Unit 1354, PO Box 301439, Houston, TX 77230-1439, USA. ${ }^{161}$ Sir Peter MacCallum Department of Oncology, Familial Cancer Centre, Peter MacCallum Cancer Centre, level 3, 10 St Andrews Place, East Melbourne, VIC 3002, Australia. ${ }^{162}$ Sir Peter MacCallum Department of Oncology, The University of Melbourne, Level 5, 161 Barry Street, Parkville 3010 VIC, Australia. ${ }^{163}$ Women's Cancer Program, Samuel Oschin Comprehensive Cancer Institute, Cedars-Sinai Medical Center, 8700 Beverly Boulevard, Los Angeles, CA 90048, USA. ${ }^{164}$ Service de génétique oncologique, Institut Curie, Inserm U830, 26 rue d'Ulm, 75248 Paris, France. ${ }^{165}$ Faculté de Médecine, Université Paris Descartes, Sorbonne Paris Cité, 15 rue de l'école de médecine, 75006 Paris, France. ${ }^{166}$ Génétique médicale, Faculté de Médecine Lyon Est, Université Claude Bernard Lyon 1, 8 avenue Rockefeller, 69373 Lyon, Cedex 08, France. ${ }^{167}$ Institut National du Cancer (INCa), La Fondation Synergie Lyon Cancer, Centre Léon Bérard, 28 rue Laënnec, 69008 Lyon, Cedex 08, France. ${ }^{168}$ Department of Laboratory Medicine and Pathology, Mayo Clinic, 200 First Street SW, Rochester, MN 55905, USA. ${ }^{169}$ Department of Cancer Epidemiology, Moffitt Cancer Center, 12902 Magnolia Drive, Tampa, FL 33612, USA. ${ }^{170}$ Unité Mixte de Génétique Constitutionnelle des Cancers Fréquents, Hospices Civils de Lyon - Centre Léon Bérard, 69373 Lyon, Cedex 08, France. ${ }^{171}$ Clinical Cancer Genetics Community Research Network, City of Hope, 1500 East Duarte Road, Duarte, CA 91010, USA.

Received: 17 September 2014 Accepted: 27 March 2015 Published online: 25 April 2015

\section{References}

1. Newman B, Austin MA, Lee M, King MC. Inheritance of human breast cancer: evidence for autosomal dominant transmission in high-risk families. Proc Natl Acad Sci U S A. 1988;85:3044-8.

2. Hall JM, Lee MK, Newman B, Morrow JE, Anderson LA, Huey B, et al. Linkage of early-onset familial breast cancer to chromosome 17q21. Science. 1990;250:1684-9.

3. Miki Y, Swensen J, Shattuck-Eidens D, Futreal PA, Harshman K, Tavtigian S, et al. A strong candidate for the breast and ovarian cancer susceptibility gene BRCA1. Science. 1994;266:66-71.

4. Evans DG, Shenton A, Woodward E, Lalloo F, Howell A, Maher ER. Penetrance estimates for BRCA1 and BRCA2 based on genetic testing in a clinical cancer genetics service setting: risks of breast/ovarian cancer quoted should reflect the cancer burden in the family. BMC Cancer. 2008;8:155.

5. Antoniou A, Pharoah PDP, Narod S, Risch HA, Eyfjord JE, Hopper JL, et al. Average risks of breast and ovarian cancer associated with BRCA1 or BRCA2 mutations detected in case Series unselected for family history: a combined analysis of 22 studies. Am J Hum Genet. 2003;72:1117-30. A published erratum appears in. Am J Hum Genet. 2003;73:709.

6. Lynch HT, Snyder C, Casey MJ. Hereditary ovarian and breast cancer: what have we learned? Ann Oncol. 2013;24 Suppl 8:viii 83-viii95.

7. Caestecker KW, Van de Walle GR. The role of BRCA1 in DNA double-strand repair: past and present. Exp Cell Res. 2013;319:575-87.

8. Negritto C. Repairing double-strand DNA breaks. Nat Educ. 2010;3:26.

9. Osorio A, Milne RL, Kuchenbaecker K, Vaclová T, Pita G, Alonso R, et al. DNA glycosylases involved in base excision repair may be associated with cancer risk in BRCA1 and BRCA2 mutation carriers. PLoS Genet. 2014;10:e1004256. 
10. Klaunig JE, Kamendulis LM, Hocevar BA. Oxidative stress and oxidative damage in carcinogenesis. Toxicol Pathol. 2010;38:96-109.

11. Loft S, Poulsen HE. Cancer risk and oxidative DNA damage in man. J Mol Med Berl. 1996;74:297-312.

12. Weng SW, Lin TK, Wang PW, Chen SD, Chuang YC, Liou CW. Single nucleotide polymorphisms in the mitochondrial control region are associated with metabolic phenotypes and oxidative stress. Gene. 2013;531:370-6.

13. Ma J, Zhang Q, Chen S, Fang B, Yang Q, Chen C, et al. Mitochondrial dysfunction promotes breast cancer cell migration and invasion through HIF1a accumulation via increased production of reactive oxygen species. PLoS One. 2013;8:e69485.

14. Kenney MC, Chwa M, Atilano SR, Falatoonzadeh P, Ramirez C, Malik D, et al. Molecular and bioenergetic differences between cells with African versus European inherited mitochondrial DNA haplogroups: implications for population susceptibility to diseases. Biochim Biophys Acta. 1842;2014:208-19.

15. Mishmar D, Ruiz-Pesini E, Golik P, Macaulay V, Clark AG, Hosseini S, et al. Natural selection shaped regional mtDNA variation in humans. Proc Natl Acad Sci U S A. 2003;100:171-6.

16. Nishimura T, Watanuki S. Relationship between mitochondrial haplogroup and seasonal changes of physiological responses to cold. J Physiol Anthropol. 2014;33:27.

17. Van der Walt JM, Dementieva YA, Martin ER, Scott WK, Nicodemus KK, Kroner CC, et al. Analysis of European mitochondrial haplogroups with Alzheimer disease risk. Neurosci Lett. 2004;365:28-32.

18. Castro MG, Huerta C, Reguero JR, Soto Ml, Doménech E, Alvarez V, et al. Mitochondrial DNA haplogroups in Spanish patients with hypertrophic cardiomyopathy. Int J Cardiol. 2006;112:202-6.

19. Malik D, Hsu T, Falatoonzadeh P, Cáceres-del-Carpio J, Tarek M, Chwa M, et al Human retinal transmitochondrial cybrids with $\mathrm{J}$ or $\mathrm{H}$ mtDNA haplogroups respond differently to ultraviolet radiation: implications for retinal diseases. PLoS One. 2014;9:e99003.

20. Mueller EE, Schaier E, Brunner SM, Eder W, Mayr JA, Egger SF, et al. Mitochondrial haplogroups and control region polymorphisms in age-related macular degeneration: a case-control study. PLoS One. 2012;7:e30874.

21. Wei L, Zhao Y, Guo T, Li P, Wu H, Xie H, et al. Association of mtDNA D-loop polymorphisms with risk of gastric cancer in Chinese population. Pathol Oncol Res. 2011;17:735-42.

22. Zhang J, Guo Z, Bai Y, Cui L, Zhang S, Xu J. Identification of sequence polymorphisms in the displacement loop region of mitochondrial DNA as a risk factor for renal cell carcinoma. Biomed Rep. 2013;1:563-6.

23. Liu WWS, Wang Y, Yang HJ, Tsang PCK, Ng TY, Wong LC, et al. Mitochondrial DNA variant 16189T $>C$ is associated with susceptibility to endometrial cancer. Hum Mutat. 2003;22:173-4.

24. Permuth-Wey J, Chen YA, Tsai YY, Chen Z, Qu X, Lancaster JM, et al. Inherited variants in mitochondrial biogenesis genes may influence epithelial ovarian cancer risk. Cancer Epidemiol Biomarkers Prev. 2011:20:1131-45.

25. Czarnecka AM, Krawczyk T, Zdrożny M, Lubiński J, Arnold RS, Kukwa W, et al. Mitochondrial NADH-dehydrogenase subunit 3 (ND3) polymorphism (A10398G) and sporadic breast cancer in Poland. Breast Cancer Res Treat. 2010;121:511-8.

26. Mims MP, Hayes TG, Zheng S, Leal SM, Frolov A, Ittmann MM, et al. Mitochondrial DNA G10398A polymorphism and invasive breast cancer in African-American women. Cancer Res. 2006;66:1880-1.

27. Tommasi S, Favia P, Weigl S, Bianco A, Pilato B, Russo L, et al. Mitochondrial DNA variants and risk of familial breast cancer: an exploratory study. Int J Oncol. 2014;44:1691-8.

28. Bahcall OG. iCOGS collection provides a collaborative model. Nat Genet. 2013:45:343.

29. Breast Cancer Association Consortium. Commonly studied single-nucleotide polymorphisms and breast cancer: results from the Breast Cancer Association Consortium. J Natl Cancer Inst. 2006:98:1382-96. A published erratum appears in J Natl Cancer Inst. 2007;99:411.

30. Berchuck A, Schildkraut JM, Pearce CL, Chenevix-Trench G, Pharoah PD. Role of genetic polymorphisms in ovarian cancer susceptibility: development of an international ovarian cancer association consortium. Adv Exp Med Biol. 2008;622:53-67.

31. Kote-Jarai Z, Easton DF, Stanford JL, Ostrander EA, Schleutker J, Ingles SA et al. Multiple novel prostate cancer predisposition loci confirmed by an international study: the PRACTICAL Consortium. Cancer Epidemiol Biomarkers Prev. 2008;17:2052-61.
32. Chenevix-Trench G, Milne RL, Antoniou AC, Couch FJ, Easton DF, Goldgar $D E$, et al. An international initiative to identify genetic modifiers of cancer risk in BRCA1 and BRCA2 mutation carriers: the Consortium of Investigators of Modifiers of BRCA1 and BRCA2 (CIMBA). Breast Cancer Res. 2007;9:104.

33. Bardel C, Danjean V, Génin E. ALTree: association detection and localization of susceptibility sites using haplotype phylogenetic trees. Bioinformatics. 2006;22:1402-3.

34. Bardel C, Danjean V, Morange P, Génin E, Darlu P. On the use of phylogeny-based tests to detect association between quantitative traits and haplotypes. Genet Epidemiol. 2009;33:729-39.

35. Couch FJ, Wang X, McGuffog L, Lee A, Olswold C, Kuchenbaecker KB, et al. Genome-wide association study in BRCA1 mutation carriers identifies nove loci associated with breast and ovarian cancer risk. PLoS Genet. 2013;9:e1003212

36. Gaudet MM, Kuchenbaecker KB, Vijai J, Klein RJ, Kirchhoff T, McGuffog L, et al. Identification of a BRCA2-specific modifier locus at $6 \mathrm{p} 24$ related to breast cancer risk. PLoS Genet. 2013;9:e1003173.

37. Center for Cancer Genetic Epidemiology, Department of Public Health and Primary Care/Department of Oncology, University of Cambridge: iCOGS custom Illumina iSelect genotyping array: access information for iCOGS data. http://ccge.medschl.cam.ac.uk/research/consortia/icogs/. Accessed 4 May 2015.

38. van Oven M, Kayser M. Updated comprehensive phylogenetic tree of global human mitochondrial DNA variation. Hum Mutat. 2009:30:E386-94.

39. Behar DM, van Oven M, Rosset S, Metspalu M, Loogväli EL, Silva NM, et al. A "Copernican" reassessment of the human mitochondrial DNA tree from its root. Am J Hum Genet. 2012;90:675-84.

40. Ge Y, Dudoit S, Speed TP. Resampling-based multiple testing for microarray data analysis. Test. 2003;12:1-77.

41. Yang Z. PAML 4: phylogenetic analysis by maximum likelihood. Mol Biol Evol. 2007;24:1586-91.

42. Antoniou AC, Goldgar DE, Andrieu N, Chang-Claude J, Brohet $R_{\text {, }}$ Rookus MA, et al. A weighted cohort approach for analysing factors modifying disease risks in carriers of high-risk susceptibility genes. Genet Epidemiol. 2005:29:1-11.

43. MITOMAP Web. HaplogroupMarkers.http://www.mitomap.org/bin/view.pl/ MITOMAP/HaplogroupMarkers. Accessed 4 May 2015.

44. Shuen AY, Foulkes WD. Inherited mutations in breast cancer genes_risk and response. J Mammary Gland Biol Neoplasia. 2011;16:3-15.

45. Jönsson G, Naylor TL, Vallon-Christersson J, Staaf J, Huang J, Ward MR, et al. Distinct genomic profiles in hereditary breast tumors identified by array-based comparative genomic hybridization. Cancer Res. 2005;65:7612-21.

46. Hughes DJ. Use of association studies to define genetic modifiers of breast cancer risk in BRCA1 and BRCA2 mutation carriers. Fam Cancer. 2008:7:233-44.

47. Antoniou AC, Sinilnikova OM, Simard J, Léoné M, Dumont M, Neuhausen SL, et al. RAD51 135G $\rightarrow C$ modifies breast cancer risk among BRCA2 mutation carriers: results from a combined analysis of 19 studies. Am J Hum Genet. 2007:81:1186-200

48. Bandelt HJ, Kloss-Brandstätter A, Richards MB, Yao YG, Logan I. The case for the continuing use of the revised Cambridge Reference Sequence (rCRS) and the standardization of notation in human mitochondrial DNA studies. J Hum Genet. 2014;59:66-77.

49. Yu X, Wieczorek S, Franke A, Yin H, Pierer M, Sina C, et al. Association of UCP2-866 G/A polymorphism with chronic inflammatory diseases. Genes Immun. 2009:10:601-5.

50. Parrella P, Xiao Y, Fliss M, Sanchez-Cespedes M, Mazzarelli P, Rinaldi M, et al. Detection of mitochondrial DNA mutations in primary breast cancer and fine-needle aspirates. Cancer Res. 2001;61:7623-6.

51. Brandon M, Baldi P, Wallace DC. Mitochondrial mutations in cancer. Oncogene. 2006;25:4647-62.

52. Bai RK, Leal SM, Covarrubias D, Liu A, Wong LC. Mitochondrial genetic background modifies breast cancer risk. Cancer Res. 2007:67:4687-94.

53. Risch HA, McLaughlin JR, Cole DEC, Rosen B, Bradley L, Fan I, et al. Population BRCA1 and BRCA2 mutation frequencies and cancer penetrances: a kin-cohort study in Ontario. Canada J Natl Cancer Inst. 2006;98:1694-706. 